\title{
Stability against contact interactions of a topological superconductor in two-dimensional space protected by time-reversal and reflection symmetries
}

\author{
Ömer M. Aksoy, ${ }_{1}^{1}$ Jyong-Hao Chen, ${ }^{2,1}$ Shinsei Ryu, ${ }^{3}$ Akira Furusaki $\odot,{ }^{4,5}$ and Christopher Mudry $\oplus^{1,6}$ \\ ${ }^{1}$ Condensed Matter Theory Group, Paul Scherrer Institute, CH-5232 Villigen PSI, Switzerland \\ ${ }^{2}$ Department of Physics, University of California, Berkeley, California 94720, USA \\ ${ }^{3}$ Department of Physics, Princeton University, Princeton, New Jersey 08540, USA \\ ${ }^{4}$ Condensed Matter Theory Laboratory, RIKEN, Wako, Saitama 351-0198, Japan \\ ${ }^{5}$ RIKEN Center for Emergent Matter Science (CEMS), Wako, Saitama 351-0198, Japan \\ ${ }^{6}$ Institut de Physique, EPF Lausanne, Lausanne CH-1015, Switzerland
}

(Received 4 February 2021; revised 23 April 2021; accepted 23 April 2021; published 13 May 2021; corrected 1 June 2021)

\begin{abstract}
We study the stability of topological crystalline superconductors in the symmetry class DIIIR and in twodimensional space when perturbed by quartic contact interactions. It is known that no less than eight copies of helical pairs of Majorana edge modes can be gapped out by an appropriate interaction without spontaneously breaking any one of the protecting symmetries. Hence, the noninteracting classification $\mathbb{Z}$ reduces to $\mathbb{Z}_{8}$ when these interactions are present. It is also known that the stability when there are less than eight modes can be understood in terms of the presence of topological obstructions in the low-energy bosonic effective theories, which prevent opening of a gap. Here, we investigate the stability of the edge theories with four, two, and one edge modes, respectively. We give an analytical derivation of the topological term for the first case, because of which the edge theory remains gapless. For two edge modes, we employ bosonization methods to derive an effective bosonic action. When gapped, this bosonic theory is necessarily associated to the spontaneous symmetry breaking of either one of time-reversal or reflection symmetry whenever translation symmetry remains on the boundary. For one edge mode, stability is explicitly established in the Majorana representation of the edge theory.
\end{abstract}

DOI: 10.1103/PhysRevB.103.205121

\section{INTRODUCTION}

Topological phases of matter have attracted ever-growing attention since the discovery of the integer quantum Hall effect in 1980 [1]. One way of understanding such phases of matter is that, in the space of gapped Hamiltonians with certain symmetries, there exist equivalence classes labeled by topological invariants. Two Hamiltonians with distinct topological invariants are topologically inequivalent since they cannot be smoothly deformed into one another without a discontinuous change of the topological invariant. In particular, a gap-closing phase transition must occur under a parametric change between two topologically distinct phases when the phase transition is continuous. If such phases of matter are short-range entangled (as opposed to long-range entangled), they are called symmetry protected topological (SPT) phases. A central theme in the study of topological matter is the classification of SPT phases.

SPT phases of noninteracting fermionic systems, which are examples of fermionic SPT (FSPT) phases, with nonspatial symmetries are well understood and described by the tenfold way [2-4]. Such phases are classified according to the spatial dimension of the physical system and the absence or presence of local nonspatial symmetries, namely, time-reversal (TR), particle hole $(\mathrm{PH})$, chiral, and their combinations. The distinctive property of these phases is that they are gapped with a nondegenerate ground state when imposing periodic boundary conditions, while they support gapless boundary states on their boundaries when imposing open boundary conditions [5]. This property is often caricatured by stating the simultaneous existence of a gap in the bulk and of gapless boundary states.

A natural extension when classifying FSPT phases is the inclusion of spatial symmetries which are relevant to crystalline materials. It has been shown that spatial symmetries such as reflection/inversion, point-group, and space-group symmetries can modify the topological classification or lead to distinct phases [6-19]. Moreover, the particular case of reflection symmetry shows that the algebra between spatial and nonspatial symmetries also affects the classification $[9,14,18,19]$. There exist 27 symmetry classes with reflection symmetries as opposed to 10 Altland-Zirnbauer symmetry classes [9].

Prior to the seminal papers in Refs. [4,20,21], it was believed that the FSPT classification was robust to symmetrypreserving local interactions. However, Fidkowski and Kitaev showed that the noninteracting boundary theory with eight zero modes for the symmetry class BDI in one-dimensional space is unstable to local and symmetry-preserving interactions by constructing such an interaction and showing that the interacting theory is adiabatically connected to a gapped boundary theory without any spontaneous breaking of the 
protecting symmetry. Hence, the noninteracting classification by group $\mathbb{Z}$ reduces to the classification by group $\mathbb{Z}_{8}$ in the presence of local interactions compatible with the BDI symmetry class in one-dimensional space. Following this work, effects of interactions have also been an important extension of the classification of noninteracting FSPT phases when local symmetry-preserving interactions are present. These effects have been investigated in Refs. [22-35].

In particular, Morimoto et al. in Ref. [28] illustrated how the topological classification for all tenfold symmetry classes and spatial dimensions can change by symmetry-preserving quartic contact interactions. They found that the noninteracting classification in terms of group $\mathbb{Z}_{2}$ is always stable to symmetry-preserving quartic contact interactions, while that with group $\mathbb{Z}$ is only stable in even spatial dimensions. For symmetry classes with the $\mathbb{Z}$ invariant in odd space dimensions, there is a breakdown to $\mathbb{Z}_{N}$ with some positive integer $N$. The method employed to show the breakdown relies on the presence or absence of topological obstructions in the low-energy bosonic theories describing $v$ copies of boundary states. These bosonic theories arise when one decouples the quartic interactions with a Hubbard-Stratonovich transformation by introducing dynamical (mass) fields and one integrates over the fermionic degrees of freedom in the path-integral description of quantum mechanics. The low-energy sector is then described by a nonlinear sigma model (NLSM) whose target space might support topological obstructions. When a local topological obstruction is present, it is conjectured in Ref. [28] that the ground state in the thermodynamic limit either remains gapless or becomes gapped with spontaneous (discrete) symmetry breaking [36]. A topological obstruction is present whenever at least one of the homotopy groups $\pi_{l}\left(\mathrm{~S}^{\mathrm{N}(v)-1}\right)$ is nontrivial [37], where $l=0, \ldots, d+1, d$ is the spatial dimension of the system, $\mathrm{S}^{\mathrm{N}(v)-1}$ is the $(\mathrm{N}(v)-1)$-dimensional sphere, and $\mathrm{N}(v)$ is the maximum number of pairwise anticommuting dynamical mass matrices for the $v$ boundary modes (the upper bound on $l$ is here dictated by locality). The number $\mathrm{N}(v)$ grows with the number of boundary modes $v$ (with plateaus when $2^{n-1}<v<2^{n}$ for some integer $n$ ). Once the inequality $\mathrm{N}(v)-1>d+1$ is satisfied, all homotopy groups $\pi_{l}\left(\mathrm{~S}^{\mathrm{N}(v)-1}\right)$ with $l=0, \ldots, d+1$ vanish so no topological obstructions compatible with locality are present. The smallest number of boundary modes $v_{\min }$ for which locality prevents the presence of a topological obstruction is conjectured in Ref. [28] to determine the breakdown pattern, i.e., the $\mathbb{Z}$ classification is reduced to the cyclic group $\mathbb{Z} \bmod$ $v_{\min }=\mathbb{Z}_{v_{\min }}$.

This strategy relying on the presence or absence of topological obstructions in NLSMs to study the effects of interactions on FSPT phases has also been applied to the crystalline topological phases. Song and Schnyder in Ref. [35] have shown the patterns for the breakdown of the noninteracting FSPT classification for all crystalline symmetry classes with reflection or twofold rotation symmetries by quartic contact interactions.

In this paper, we focus on TR symmetric two-dimensional crystalline topological superconductors. They realize the FSPT phase DIII. In addition to TR symmetry (TRS), we impose reflection symmetry (RS) and denote with DIIIR a superconducting phase protected by TRS, RS, and translation symmetry (TS). The noninteracting FSPT of symmetry class DIII in two spatial dimensions has a $\mathbb{Z}_{2}$ group structure. The noninteracting FSPT of symmetry class DIIIR in two spatial dimensions has a $\mathbb{Z}$ group structure when reflection has a unitary Hermitian representation that anticommutes with the representations of both reversal of time and the interchange of particles and holes [9,22,38].

The interacting classification of the FSPT symmetry class DIIIR in two-dimensional space was first obtained in Ref. [22] by showing that no less than eight copies of the gapless edge theory can be gapped without spontaneous breaking of the protecting symmetries by local interactions. Hence, local and symmetry-preserving interactions reduce the noninteracting classification with group $\mathbb{Z}$ to the one with group $\mathbb{Z}_{8}$. The same result was later obtained in Refs. $[28,35]$ by showing on general grounds the possibility for the existence of topological obstructions in the NLSM description of the edge theory using homotopy arguments. However, it remains open to (i) explicitly construct these topological obstructions in the low-energy theory and to (ii) explicitly show how the gapless edge modes remain stable in the presence of local and symmetry-preserving interactions because of the topological obstructions.

We start from an FSPT symmetry class DIIIR in two spatial dimensions and aim to derive its low-energy bosonic action along one of its connected boundaries explicitly. The fermionic edge theory is described by $v$ copies of helical Majorana fields. If interactions are absent, this edge theory cannot be gapped, whatever the value of $v \in \mathbb{Z}$. If quartic contact interactions are present, Table I implies the existence of a topological obstruction when $v=1$, another one when $v=2,3$, and the last one when $v=4,5,6,7$. The goal of this paper is to construct explicitly these topological obstructions. To achieve this end, it is sufficient to start from an interacting boundary Hamiltonian for $v=1, v=2$, and $v=4$ copies of relativistic helical Majorana fields, respectively [28].

There are three possibilities when local interactions that preserve the TRS, RS and TS are included: (i) the edge theory remains gapless, (ii) the edge theory is gapped but some of the protecting symmetries are spontaneously broken, and (iii) the edge theory is gapped without any symmetry breaking. We call the edge theory unstable against the given interactions when the last scenario is realized, otherwise we say that it is stable.

Out of three cases $(v=1,2,4)$ considered, we derive the topological obstruction (i.e., the topological term in the NLSM) for $v=4$ case explicitly via the gradient expansion of a fermionic determinant. We identify $\mathrm{N}(4)=4$ possible quartic interactions which leads to the nontrivial homotopy group $\pi_{3}\left(S^{3}\right)=\mathbb{Z}$. The corresponding term is of the WessZumino (WZ) type, as is indicated by line $D=4$ and column 4 from Table I. It is expected that NLSM action supplemented with an appropriate WZ term is equivalent to an action for fermions in $(1+1)$ dimensions at criticality [39]. Hence, the edge theory remains stable.

For the remaining two cases, the method of gradient expansion turns out to be difficult to apply. We identify $\mathrm{N}(2)=$ 2 and $\mathrm{N}(1)=1$ interaction channels for two and one edge modes, respectively. In the former case, $\pi_{1}\left(S^{1}\right)=\mathbb{Z}$ implies 
TABLE I. Reduction from $\mathbb{Z}$ to $\mathbb{Z}_{8}$ due to interactions for the topologically equivalent classes of the two-dimensional topological superconductors protected by time-reversal, reflection, and translation symmetries (DIIIR). If $V_{v}$ denotes the space of $v \times v$ normalized dynamical Dirac mass matrices on a boundary invariant under both reflection and translation, then the limit $v \rightarrow \infty$ of these spaces is the classifying space $R_{1}$ [28]. The second column shows the stable $D$ th homotopy groups of the classifying space $R_{1}$. The third column gives the number $v$ of copies of boundary (Dirac) fermions for which a topological obstruction is permissible. The fourth column gives the type of topological obstruction that prevents the gapping of the boundary (Dirac) fermions. We note that for $D=7$, even though the $\pi_{7}\left(R_{1}\right)$ is nonvanishing, there is no topological obstruction that is compatible with locality. The topological obstruction for $v=2$ is also present when $v=3$ and the topological obstruction for $v=4$ is also present when $v=5,6,7$.

\begin{tabular}{cccc}
\hline \hline$D$ & $\pi_{D}\left(R_{1}\right)$ & $v$ & Topological obstruction \\
\hline 0 & $\mathbb{Z}_{2}$ & 1 & Domain wall \\
1 & $\mathbb{Z}_{2}$ & 2 & Vortex \\
2 & 0 & & WZ term \\
3 & $\mathbb{Z}$ & 4 & \\
4 & 0 & & None \\
5 & 0 & & \\
6 & 0 & 8 & \\
7 & $\mathbb{Z}$ & & \\
\hline \hline
\end{tabular}

the existence of vortex configurations of the NLSM bosonic field, as indicated by line $D=1$ and column 4 from Table I. In the latter case, $\pi_{0}\left(\mathrm{~S}^{0}\right)=\mathbb{Z}_{2}$ implies the existence of domainwall configurations, as indicated by line $D=0$ and column 4 from Table I. However, vortex configurations invalidate the gradient expansion by being singular at the vortex core and domain-wall configurations necessarily bind zero energy modes, which leads to vanishing fermionic determinants.

For the $v=2$ case, we follow an alternative approach by adopting bosonization techniques. The low-energy edge theory turns out to be described by a sine-Gordon action. Such a theory is not necessarily gapless; however, we demonstrate that any twofold degenerate and translation-invariant gapped ground state spontaneously breaks either the TRS or the RS. This analysis is then supplemented by considering interactions that, prior to bosonization, are local momonials in certain fermionic bilinears of arbitrary order (i.e., not necessarily of quadratic order). We find that spontaneous symmetry breaking of either the TRS or RS always occurs when the interaction is strong and of even order, while these protecting symmetries are not broken but TS is explicitly broken when the interaction is strong and of odd order. In the latter case, there are no protected delocalized edge states within the bulk gap, but there are "corner" states on the boundary within the bulk gap.

For the case of $v=1$, we consider smooth interpolations between certain mass profiles and show the existence of two topological sectors distinguished by a $\mathbb{Z}_{2}$ invariant. The existence of these two topological sectors is closely related to a global $\mathbb{Z}_{2}$ anomaly.

The paper is organized as follows. In Sec. II, we define the boundary Hamiltonian for $v$-edge modes and the symmetries of the class DIIIR. The subsequent sections discusses the cases $v=4,2,1$, respectively. We conclude with Sec. VI.

\section{DEFINITIONS AND SYMMETRIES}

We describe the one-dimensional boundary of a twodimensional crystalline topological superconductor with the Hamiltonian

$$
\begin{aligned}
\widehat{H}_{\mathrm{bd}} & :=\widehat{H}_{0}+\widehat{H}_{\mathrm{int}}, \\
\widehat{H}_{0} & \equiv \int d x \hat{\chi}^{\dagger} \mathcal{H}_{0} \hat{\chi} \\
& :=\int d x \hat{\chi}^{\dagger}\left(\sigma_{3} \otimes \mathbb{1}_{v} \mathrm{i} \partial_{x}\right) \hat{\chi}, \\
\widehat{H}_{\mathrm{int}} & =-\int d x \lambda^{2} \sum_{l=1}^{\mathrm{N}(v)}\left(\hat{\chi}^{\dagger} \beta_{l} \hat{\chi}\right)^{2} .
\end{aligned}
$$

The Hamiltonian $\widehat{H}_{0}$ describes $v$ pairs of left- and rightmoving quantum Majorana fields. The components $\hat{\chi}_{a}$ and $\hat{\chi}_{a}^{\dagger}$ with $a=1, \ldots, 2 v$ of the quantum-fields and their adjoints obey the equal-time algebra

$$
\left\{\hat{\chi}_{a}(x), \hat{\chi}_{a^{\prime}}\left(x^{\prime}\right)\right\}=\delta_{a a^{\prime}} \delta\left(x-x^{\prime}\right),
$$

with all other anticommutators vanishing, and we impose the Majorana condition

$$
\hat{\chi}^{\dagger}=\hat{\chi}^{\top}
$$

The Hamiltonian $\widehat{H}_{\text {int }}$ encodes the quartic contact interactions with coupling constant $\lambda^{2}$ between the $v$ different flavors. The matrix $\mathbb{1}_{v}$ is the identity matrix in flavor space. The label $l=1, \ldots, \mathrm{N}(v)$ enumerates all $2 v \times 2 v$ Hermitian matrices such that (i) they square to the identity $\beta_{l}^{2}=\mathbb{1}_{2 v}$, (ii) any pair $\left(\beta_{l}, \beta_{l^{\prime}}\right)$ anticommutes pairwise as well as with $\sigma_{3} \otimes \mathbb{1}_{v}$, and (iii) each $\beta_{l}$ is odd under complex conjugation. The first two conditions restrict the $\mathrm{N}(v)$ interaction channels to the squares of bilinears that are not competing Dirac mass terms. The last condition follows from imposing a Majorana condition on the fermionic quantum fields as we do now. Had we demanded instead of (iii) that each $\beta_{l}$ is even under complex conjugation, the bilinear $\hat{\chi}^{\dagger} \beta_{l} \hat{\chi}$ would then vanish because of the Majorana condition. We emphasize that $\mathrm{N}(v)$ is constant when $2^{n-1}<v<2^{n}$ for some integer $n$. This means that the target space corresponding to the normalized dynamical Dirac masses does not change when $2^{n-1}<v<2^{n}$ for some integer $n$.

Following Refs. [9,22], we define the PH, TR, and reflection transformations,

$$
\begin{aligned}
\mathcal{C}_{\mathrm{bd}, v} & :=\mathbb{1}_{2} \otimes \mathbb{1}_{\nu} \mathrm{K}, \\
\mathcal{T}_{\mathrm{bd}, v} & :=\mathrm{i} \sigma_{2} \otimes \mathbb{1}_{\nu} \mathrm{K}, \\
\mathcal{R}_{\mathrm{bd}, v} & :=\mathrm{i} \sigma_{2} \otimes \mathbb{1}_{\nu},
\end{aligned}
$$

where $\sigma$ are the Pauli matrices and $\mathrm{K}$ denotes complex conjugation. They satisfy the defining conditions of the symmetry 
class DIIIR, i.e.,

$$
\mathcal{C}_{\mathrm{bd}, v}^{2}=+1, \quad \mathcal{T}_{\mathrm{bd}, \nu}^{2}=-1, \quad \mathcal{R}_{\mathrm{bd}, v}^{2}=-1,
$$

with the algebra

$$
\left[\mathcal{C}_{\mathrm{bd}, v}, \mathcal{R}_{\mathrm{bd}, v}\right]=0, \quad\left[\mathcal{T}_{\mathrm{bd}, \nu}, \mathcal{R}_{\mathrm{bd}, \nu}\right]=0 .
$$

Had we chosen the Hermitian representation for the reflection transformation (2.3c), i.e., $\mathcal{R}_{\mathrm{bd}, v}=\sigma_{2} \otimes \mathbb{1}_{\nu}$, it would anticommute with both $\mathrm{PH}$ and TR transformations. This is consistent with the definition of DIIIR in Ref. [9]. The anti-Hermitian representation (2.3c) is chosen since the transformation law is then covariant with respect to the Majorana condition (2.2b). Moreover, we demand that transformations (2.3) are (spectral) symmetries of the single-particle Hamiltonian $(2.1 \mathrm{~b})$

$$
\begin{aligned}
\mathcal{C}_{\mathrm{bd}, v} \mathcal{H}_{0}(x) \mathcal{C}_{\mathrm{bd}, v}^{-1} & =-\mathcal{H}_{0}(x), \\
\mathcal{T}_{\mathrm{bd}, \nu} \mathcal{H}_{0}(x) \mathcal{T}_{\mathrm{bd}, \nu}^{-1} & =+\mathcal{H}_{0}(x), \\
\mathcal{R}_{\mathrm{bd}, v} \mathcal{H}_{0}(-x) \mathcal{R}_{\mathrm{bd}, \nu}^{-1} & =+\mathcal{H}_{0}(x) .
\end{aligned}
$$

When the conditions (2.6) are satisfied and we impose invariance under TS,

$$
\widehat{T}\left(x^{\prime}\right) \widehat{H}_{\mathrm{bd}} \widehat{T}^{-1}\left(x^{\prime}\right)=\widehat{H}_{\mathrm{bd}}, \quad \forall x^{\prime} \in \mathbb{R},
$$

where $\widehat{T}\left(x^{\prime}\right)$ is the operator that implements the translation by $x^{\prime}$, then Hamiltonian (2.1b) cannot be gapped by adding bilinears of the fermionic fields for any $v=1,2,3, \ldots$ [9]. In this case, the noninteracting classification for the class DIIIR is $\mathbb{Z}$. However, bilinears that are odd under reflection are allowed if they are multiplied by a (smooth) function of $x$, a space-dependent mass, that is odd under $x \rightarrow-x$ and must thus vanish at the origin $x=0$. Such a mass gaps the single-particle spectrum except for a mid-gap bound state whose envelope decays exponentially fast away from $x=0$. Any such mass breaks TS and the origin can be thought of as a point defect or a "corner" along the one-dimensional boundary at which the mass term must change sign if it is to respect reflection symmetry. In the presence of such a spacedependent mass, the noninteracting classification reduces to that of the symmetry class DIII, i.e., $\mathbb{Z}_{2}[9,22,40]$.

Alternatively, we can write down the partition function

$$
\begin{aligned}
Z_{\mathrm{bd}} & :=\int \mathcal{D}[\chi] e^{-S_{\mathrm{bd}}}, \\
S_{\mathrm{bd}} & :=\int d \tau d x \mathcal{L}_{\mathrm{bd}}, \\
\mathcal{L}_{\mathrm{bd}} & :=\chi^{\dagger}\left(\partial_{\tau}+\sigma_{3} \otimes \mathbb{1}_{\nu} \mathrm{i} \partial_{x}\right) \chi-\lambda^{2} \sum_{l=1}^{\mathrm{N}(v)}\left(\chi^{\dagger} \beta_{l} \chi\right)^{2},
\end{aligned}
$$

where the action is defined on $(1+1)$-dimensional Euclidean space-time. The integration variables are the components of the Grassmann-valued spinor $\chi$, as $\chi^{\dagger}$ is linearly constrained to $\chi$ through the Majorana condition (2.2b). The interaction terms can be decoupled via Hubbard-Stratonovich transformation. The partition function (2.8) then takes the form

$$
\begin{aligned}
Z_{\mathrm{bd}} & =\text { const } \times \int \mathcal{D}[\chi] \int \mathcal{D}\left[\phi_{\beta_{l}}\right] e^{-S_{\mathrm{bd}}^{\prime}}, \\
S_{\mathrm{bd}}^{\prime} & =\int d \tau d x \mathcal{L}_{\mathrm{bd}}^{\prime}, \\
\mathcal{L}_{\mathrm{bd}}^{\prime} & =\chi^{\dagger}\left(\partial_{\tau}+\mathcal{H}_{\mathrm{bd}}^{(\mathrm{dyn})}\right) \chi+\frac{1}{(2 \lambda)^{2}} \sum_{l=1}^{\mathrm{N}(v)} \phi_{l}^{2}, \\
\mathcal{H}_{\mathrm{bd}}^{(\mathrm{dyn})} & :=+\sigma_{3} \otimes \mathbb{1}_{v} \mathrm{i} \partial_{x}+\sum_{l=1}^{\mathrm{N}(\nu)} \beta_{l} \phi_{l} .
\end{aligned}
$$

We have thereby defined the dynamical single-particle boundary Hamiltonian $\mathcal{H}_{\mathrm{bd}}^{(\mathrm{dyn})}$. Conditions (2.6) on $\mathcal{H}_{\mathrm{bd}}^{(\mathrm{dyn})}$ can be met as follows. PHS imposes that

$$
\mathrm{K} \beta_{l} \mathrm{~K}^{-1}=\beta_{l}^{*}=-\beta_{l}, \quad l=1, \ldots, \mathrm{N}(v)
$$

for any $\beta_{l}$ Hermitian $2 v \times 2 v$ matrix. Hence, imposing the Majorana condition trivially satisfies the PHS. Once the maximum number of $\beta_{l}$ matrices that are compatible with PHS is found, the symmetry requirements coming from TRS and RS can be satisfied by imposing that $\phi_{l}$ is either odd or even under time-reversal and reflection. From now on, we shall use the shorthand notation for the $4^{n}, 2^{n} \times 2^{n}$ Hermitian matrices

$$
\begin{aligned}
\mathrm{X}_{\mu_{1} \mu_{2} \ldots \mu_{n}} & :=\sigma_{\mu_{1}}^{(1)} \otimes \sigma_{\mu_{2}}^{(2)} \otimes \sigma_{\mu_{3}}^{(3)} \otimes \cdots \otimes \sigma_{\mu_{n}}^{(n)}, \\
\left(\mathrm{X}_{\mu_{1} \mu_{2} \ldots \mu_{n}}\right)^{2} & =\mathbb{1}_{2^{n}}, \quad \mu_{j}=0,1,2,3,
\end{aligned}
$$

where $\sigma_{0}^{(j)}$ is $\mathbb{1}_{2}, \boldsymbol{\sigma}^{(j)}$ are the associated Pauli matrices and $n \in \mathbb{Z}$ is related to $v$ by the relation $2^{n-1}=v$.

The partition function (2.9) is quadratic in Grassmann variables, which therefore can be integrated out to yield an effective action of bosonic fields $\phi_{\beta_{l}}$, provided the Majorana Pfaffian is nonvanishing. This effective theory is described by the partition function

$$
Z=\int \mathcal{D}[\boldsymbol{\phi}] \delta\left(\boldsymbol{\phi}^{2}-\bar{\phi}^{2}\right) e^{-\int d^{2} x \frac{1}{g}\left(\partial_{\mu} \phi\right)^{2}+\Gamma[\boldsymbol{\phi}]},
$$

where $\bar{\phi}^{2}>0$ is a real-valued constant, $\boldsymbol{\phi}$ is a $\mathrm{N}(v)$ dimensional vector field that is normalized through the nonlinear constraint imposed by the $\delta$ function, and the symbol $\Gamma[\phi]$ signifies the existence of a topological obstruction. In other words, the presence of the symbol $\Gamma[\phi]$ implies that the effective action associated to the partition function (2.12) is not merely that of a NLSM. Due to the nonlinear constraint imposed on $\mathrm{N}(v)$ bosonic fields, the target space in Eq. (2.12) is the unit sphere $S^{\mathrm{N}(v)-1}$. The symbol $\Gamma[\phi]$ is present in Eq. (2.12) whenever one of the homotopy groups,

$$
\begin{aligned}
& \pi_{0}\left(\mathrm{~S}^{\mathrm{N}(v)-1}\right), \\
& \pi_{1}\left(\mathrm{~S}^{\mathrm{N}(v)-1}\right), \\
& \pi_{2}\left(\mathrm{~S}^{\mathrm{N}(v)-1}\right), \\
& \cdots \\
& \pi_{d+1}\left(\mathrm{~S}^{\mathrm{N}(v)-1}\right),
\end{aligned}
$$

is nontrivial [37]. (The upper bound $d+1$ is imposed as topological obstructions corresponding to higher homotopy 
groups modify the equations of motions in a nonlocal way [28].) Such topological obstructions are expected to prevent gapping out the edge modes. If no such topological obstructions exists, the low-energy effective theory is described by no more than a NLSM action. For space-time dimension two, the action then flows to the strong coupling $g \rightarrow \infty$ stable fixed point [41]. This is the quantum disordered phase that describes a gapped phase of matter that is symmetric under all protecting symmetries. The original $v$ gapless edge modes have been gapped by the interactions without any of the preserving symmetries being spontaneously broken. Hence, the noninteracting gapless edge theory is smoothly connected to a strongly interacting gapped edge theory upon switching on local symmetry-preserving interactions. The presence of the topological obstruction manifests itself by modifying the renormalization group (RG) flow and preventing the flow to the strong coupling limit $g \rightarrow \infty$.

\section{THE CASE $v=4$}

The set (2.11) with $n=3$ has the 64 elements $\left\{\mathrm{X}_{\mu \rho \sigma}\right\}$ with $\mu, \rho, \sigma=0, \ldots, 3$. This set spans the space of $8 \times 8$ Hermitian matrices. For $v=4$, there are at most $\mathrm{N}(4)=4$ interaction channels allowed by the symmetry conditions (2.6), each of which is labeled by the Hermitian $8 \times 8$ matrix $\beta_{l}$. We consider the parametrization

$$
\mathcal{H}_{\text {bd }}^{\mathrm{dyn}}(\tau, x):=\beta_{0} \mathrm{i} \partial_{x}+\sum_{l=1}^{4} \beta_{l} \phi_{l}(\tau, x)
$$

of the dynamical boundary single-particle Hamiltonian, where without loss of generality, we make the choice

$$
\begin{aligned}
& \beta_{0}:=X_{300}, \\
& \beta_{1}:=X_{210}, \\
& \beta_{2}:=X_{230}, \\
& \beta_{3}:=X_{222}, \\
& \beta_{4}:=X_{102}=-X_{300} X_{210} X_{230} X_{222} .
\end{aligned}
$$

The choice $\left\{\beta_{1}, \beta_{2}, \beta_{3}, \beta_{4}\right\}$ is not unique but this lack of uniqueness does not affect the subsequent analysis. We define the corresponding partition function

$$
\begin{aligned}
Z_{\mathrm{bd}} & :=\int \mathcal{D}[\chi] \int \mathcal{D}[\Phi] e^{-S_{\mathrm{bd}}}, \\
S_{\mathrm{bd}} & :=\int d^{2} x\left[\bar{\chi}\left(\mathrm{i} \gamma_{\mu} \partial_{\mu}+\Phi\right) \chi+\frac{1}{(2 \lambda)^{2}} \Phi^{\dagger} \Phi\right],
\end{aligned}
$$

where, following Ref. [42], we have introduced the notations

$$
\begin{aligned}
\bar{\chi} & :=\chi^{\dagger}\left(-\mathrm{i} \gamma_{0}\right), \\
\gamma_{0} & :=\beta_{4}=\mathrm{X}_{102}, \\
\gamma_{1} & :=\mathrm{i} \beta_{4} \beta_{0}=\mathrm{X}_{202}, \\
\gamma_{5} & :=\gamma_{0} \gamma_{1}=\mathrm{i} \beta_{0}=\mathrm{i} \mathrm{X}_{300}, \\
\Upsilon_{1} & :=-\mathrm{X}_{312}, \\
\Upsilon_{2} & :=-\mathrm{X}_{332}, \\
\Upsilon_{3} & :=-\mathrm{X}_{320}, \\
\Upsilon_{4} & :=+\mathrm{iX}_{000},
\end{aligned}
$$

and have defined the matrix-valued field

$$
\begin{aligned}
& \Phi(x):=|\boldsymbol{\phi}(x)| \sum_{l=1}^{4} n_{l}(x) \Upsilon_{l}, \\
& \boldsymbol{\phi}(x):=|\boldsymbol{\phi}(x)| \boldsymbol{n}(x) \in \mathbb{R}^{4}, \quad \boldsymbol{n}^{2}(x)=1,
\end{aligned}
$$

that parametrizes the dynamical mass profile. We denote the imaginary time and space coordinates by $x=\left(x_{0}, x_{1}\right) \equiv$ $(\tau, x)$. With the choice of the representation made in Eqs. (3.2), the identities

$$
\left\{\gamma_{\mu}, \gamma_{\nu}\right\}=2 \delta_{\mu \nu}, \quad\left\{\gamma_{\mu}, \Upsilon_{l}\right\}=2 \delta_{4 l} \Upsilon_{l} \gamma_{\mu},
$$

hold for any $\mu, v=0,1$ and $l=1,2,3,4$. Performing the Grassmann integration on the partition function (3.2) delivers the bosonic and local effective action

$$
\begin{aligned}
Z_{\text {eff }} & :=\int \mathcal{D}[\Phi] e^{-S_{\text {eff }}[\Phi]} \\
S_{\text {eff }}[\Phi] & :=-\frac{1}{2} \operatorname{Tr}\left[\ln \mathcal{D}_{\Phi}\right]+\frac{1}{32 \lambda^{2}} \operatorname{Tr}\left[\Phi^{\dagger} \Phi\right] \\
\mathcal{D}_{\Phi} & :=\mathrm{i} \gamma_{\mu} \partial_{\mu}+\Phi
\end{aligned}
$$

Here, the trace $\mathrm{Tr}$ is understood to be over both a plane-wave basis and $8 \times 8$ matrices. The local effective action (3.4b) can be written in closed form to any finite order of a gradient expansion [37] as we now sketch.

The solution $\bar{\Phi}$ to the saddle-point equation

$$
\frac{\delta S_{\text {eff }}}{\delta \Phi}=0
$$

is

$$
\bar{\Phi}=\bar{\phi} \sum_{\iota=1}^{4} \bar{n}_{\iota} \Upsilon_{\iota}
$$

where

$$
\sum_{\iota=1}^{4} \bar{n}_{\iota}^{2}=1, \quad \bar{\phi}^{2}:=\left(e^{\frac{1}{8 \pi \lambda^{2}}}-1\right)^{-1} \Lambda^{2} .
$$

Here, $\Lambda$ is the UV cutoff introduced to regularize the integration over momenta. The direction of the saddle-point solution $\overline{\boldsymbol{n}}$ is arbitrary.

Next, we first consider the change $\delta S_{\text {eff }}[\Phi]$ of effective action (3.4) when $\Phi$ is varied to $\Phi+\delta \Phi$,

$$
\delta S_{\text {eff }}[\Phi]=S_{\text {eff }}[\Phi+\delta \Phi]-S_{\text {eff }}[\Phi],
$$

which is to be expanded around the saddle-point solution (3.5) in powers of $1 / \bar{\phi}^{2}$. Taking the limit $\bar{\phi}^{2} \rightarrow \infty$ kills all but a finite number of terms on the right-hand side of Eq. (3.6). Integration over $\delta \Phi$ then delivers two terms. The first term is

$$
S_{\mathrm{NLSM}}=\int d^{2} x \frac{1}{2 g}\left(\partial_{\mu} n\right)^{2}, \quad g=\pi .
$$

This is the action of the O(4)-NLSM in two-dimensional Euclidean space-time with the bare coupling constant $g=\pi$. The second term is

$$
\Gamma=\frac{2 \mathrm{i} \pi}{3 ! \operatorname{Area}\left(\mathrm{S}^{3}\right)} \int d^{3} \tilde{x} \epsilon_{\mu \nu \rho} \epsilon_{a b c d}\left(\partial_{\mu} \tilde{n}_{a}\right)\left(\partial_{\nu} \tilde{n}_{b}\right)\left(\partial_{\rho} \tilde{n}_{c}\right) \tilde{n}_{d} .
$$


Indeed, imposing the nonlinear constraint $\boldsymbol{n}^{2}(x)=1$ compactifies the target space of the O(4)-NLSM to the three-sphere $\mathrm{S}^{3}$. This sphere has the nontrivial homotopy group $\pi_{3}\left(\mathrm{~S}^{3}\right)=$ $\mathbb{Z}$. It is then meaningful following Witten [39] to introduce an auxiliary coordinate $u \in[0,1]$ and to extend the domain of definition of the field $\boldsymbol{n}(x)$ from $\mathbb{R}^{2}$ to $\mathbb{R}^{2} \times[0,1]$, $\boldsymbol{n}(x) \rightarrow \tilde{\boldsymbol{n}}(x, u), d^{2} x \rightarrow d^{2} x d u \equiv d^{3} \tilde{x}$ such that the boundary conditions $\overline{\boldsymbol{n}}(x, 0)=\boldsymbol{n}_{0}$ for some arbitrary direction $\boldsymbol{n}_{0}$ and $\tilde{\boldsymbol{n}}(x, 1)=\boldsymbol{n}(x)$ are satisfied. This is the WZ [39,43-45] term for the O(4)-NLSM in two-dimensional Euclidean spacetime. This term is not local in the action but its effect on the equations of motion is local. However, this term modifies nonperturbatively the RG flow obeyed by the coupling $g$. In fact, in the presence of the WZ term, the beta function of $g$ has been conjectured to vanish at the value $g_{\mathrm{c}}=\pi$ that defines a critical point with conformal symmetry $[37,39]$.

The interaction that we chose has an $\mathrm{O}(4)$ symmetry. This symmetry is not sacred. For example, we could have introduced four dimensionless couplings $\lambda_{l}$ with $l=1, \ldots, 4$, one for each dynamical mass $\beta_{l}$ in Eqs. (3.1). By treating each dynamical mass $\beta_{l}$ as independent Hubbard-Stratonovich fields and integrating over these fields, the interaction is the sum of four quartic contact interactions, each of which is weighted by the multiplicative factor $\left(2 \lambda_{l}\right)^{-2}$. This interacting theory can be bosonized with the help of Abelian bosonization rules. The stability analysis then proceeds along the same line as what is done in Sec. IV B with the same conclusions. The boundary theory is gapped if and only if the protecting symmetries (2.6) or (2.7) are spontaneously broken. One may repeat this exercise with $v=6$ and reach the same conclusion, a gap is necessarily associated with the spontaneous symmetry breaking of the TRS or RS. It is only when $v$ is an integer multiple of the number 8 that a gap delivers a nondegenerate ground state.

\section{THE CASE $v=2$}

The set (2.11) with $n=2$ has the 16 elements $\left\{\mathrm{X}_{\mu \rho}\right\}$ with $\mu, \rho=0, \ldots, 3$. This set spans the space of $4 \times 4$ Hermitian matrices. For $v=2$, there are at most $\mathrm{N}(2)=2$ interaction channels allowed by the symmetry conditions (2.6), each of which is labeled by the Hermitian $4 \times 4$ matrix $\beta_{l}$. We consider the parametrization

$$
\mathcal{H}_{\mathrm{bd}}^{(\mathrm{dyn})}(\tau, x):=\beta_{0} \mathrm{i} \partial_{x}+\sum_{l=1}^{2} \beta_{l} \phi_{l}(\tau, x)
$$

of the dynamical boundary single-particle Hamiltonian. Following the same steps as in Sec. III, we impose the nonlinear constraint:

$$
\phi_{1}^{2}(\tau, x)+\phi_{2}^{2}(\tau, x)=\bar{\phi}^{2} .
$$

This condition compactifies the target space of the effective bosonic and local theory to the circle $S^{1}$. However, the nontrivial fundamental group $\pi_{1}\left(S^{1}\right)=\mathbb{Z}$ implies the existence of a topological obstruction. This topological obstruction takes the form of point defects when the vector $\left(\phi_{1}(\tau, x), \phi_{2}(\tau, x)\right)$ accommodates vortex configurations in $(1+1)$-dimensional space-time. A vortex configuration is singular at the vortex core where its gradient is ill-defined.
Direct application of the gradient expansion method employed in Sec. III is thus invalid. To circumvent this difficulty, we choose the method of Abelian bosonization to derive an effective local bosonic action.

\section{A. Abelian bosonization}

We start from

$$
\widehat{H}_{\mathrm{bd}}:=\int d x\left\{\hat{\chi}^{\dagger} \mathrm{X}_{30} \mathrm{i} \partial_{x} \hat{\chi}-\sum_{l=1}^{2} \lambda_{l}^{2}\left(\hat{\chi}^{\dagger} \beta_{l} \hat{\chi}\right)^{2}\right\},
$$

i.e., we do not impose the $\mathrm{O}(2)$ symmetry resulting from demanding that $\lambda_{1}^{2}=\lambda_{2}^{2}=\lambda^{2}$ as is done in Hamiltonian (2.1). Imposing symmetry conditions (2.6a) leads to the identification of two possible sets $\left\{\beta_{l}\right\}$ :

$$
\mathcal{B}_{a}=\left\{\mathrm{X}_{12}, \mathrm{X}_{20}\right\}, \quad \mathcal{B}_{b}=\left\{\mathrm{X}_{21}, \mathrm{X}_{23}\right\}
$$

Choosing set $\mathcal{B}_{a}$ in Eq. (4.3) defines $\widehat{H}_{\mathrm{bd} a}$. Choosing set $\mathcal{B}_{b}$ in Eq. (4.3) defines $\widehat{H}_{\mathrm{bd} b}$. We will perform the subsequent analysis for both $\widehat{H}_{\mathrm{bd} a}$ and $\widehat{H}_{\mathrm{bd} b}$ in parallel. With the convention

$$
\hat{\chi}^{\dagger}=\left(\hat{\chi}_{\mathrm{L}}^{1}, \hat{\chi}_{\mathrm{L}}^{2}, \hat{\chi}_{\mathrm{R}}^{1}, \hat{\chi}_{\mathrm{R}}^{2}\right),
$$

Hamiltonians $\widehat{H}_{\mathrm{bd} a}$ and $\widehat{H}_{\mathrm{bd} b}$ are given by

$$
\begin{aligned}
\widehat{H}_{\mathrm{bd} a}:= & \int d x\left\{\hat{\chi}^{\dagger} \mathrm{X}_{30} \mathrm{i} \partial_{x} \hat{\chi}\right. \\
& \left.-\lambda_{1, a}^{2}\left(\hat{\chi}^{\dagger} \mathrm{X}_{12} \hat{\chi}\right)^{2}-\lambda_{2, a}^{2}\left(\hat{\chi}^{\dagger} \mathrm{X}_{20} \hat{\chi}\right)^{2}\right\}
\end{aligned}
$$

and

$$
\begin{aligned}
\widehat{H}_{\mathrm{bd} b}:= & \int d x\left\{\hat{\chi}^{\dagger} \mathrm{X}_{30} \mathrm{i} \partial_{x} \hat{\chi}\right. \\
& \left.-\lambda_{1, b}^{2}\left(\hat{\chi}^{\dagger} \mathrm{X}_{21} \hat{\chi}\right)^{2}-\lambda_{2, b}^{2}\left(\hat{\chi}^{\dagger} \mathrm{X}_{23} \hat{\chi}\right)^{2}\right\},
\end{aligned}
$$

respectively. This Majorana representation is not well suited for Abelian bosonization. Instead of it, we define the rightmoving complex fermion fields

$$
\hat{\psi}_{\mathrm{R}}^{\dagger}:=\frac{\hat{\chi}_{\mathrm{R}}^{1}-\mathrm{i} \hat{\chi}_{\mathrm{R}}^{2}}{\sqrt{2}}, \quad \hat{\psi}_{\mathrm{R}}:=\frac{\hat{\chi}_{\mathrm{R}}^{1}+\mathrm{i} \hat{\chi}_{\mathrm{R}}^{2}}{\sqrt{2}},
$$

the left-moving complex fermion fields

$$
\hat{\psi}_{\mathrm{L}}^{\dagger}:=\frac{\hat{\chi}_{\mathrm{L}}^{1}-\mathrm{i} \hat{\chi}_{\mathrm{L}}^{2}}{\sqrt{2}}, \quad \hat{\psi}_{\mathrm{L}}:=\frac{\hat{\chi}_{\mathrm{L}}^{1}+\mathrm{i} \hat{\chi}_{\mathrm{L}}^{2}}{\sqrt{2}},
$$

and the complex fermion basis

$$
\hat{\Psi}^{\dagger}=\left(\begin{array}{llll}
\hat{\psi}_{\mathrm{L}}^{\dagger} & \hat{\psi}_{\mathrm{R}}^{\dagger} & \hat{\psi}_{\mathrm{L}} & \hat{\psi}_{\mathrm{R}}
\end{array}\right)
$$

In the basis (4.6c), we find the complex fermion representation

$$
\begin{aligned}
\widehat{H}_{\mathrm{bd} a}:= & \int d x\left\{\hat{\Psi}^{\dagger} \mathrm{X}_{03} \mathrm{i} \partial_{x} \hat{\Psi}\right. \\
& \left.-\lambda_{1, a}^{2}\left(\hat{\Psi}^{\dagger} \mathrm{X}_{31} \hat{\Psi}\right)^{2}-\lambda_{2, a}^{2}\left(\hat{\Psi}^{\dagger} \mathrm{X}_{02} \hat{\Psi}\right)^{2}\right\}
\end{aligned}
$$

and

$$
\begin{aligned}
\widehat{H}_{\mathrm{bd} b}:= & \int d x\left\{\hat{\Psi}^{\dagger} \mathrm{X}_{03} \mathrm{i} \partial_{x} \hat{\Psi}\right. \\
& \left.-\lambda_{1, b}^{2}\left(\hat{\Psi}^{\dagger} \mathrm{X}_{22} \hat{\Psi}\right)^{2}-\lambda_{2, b}^{2}\left(\hat{\Psi}^{\dagger} \mathrm{X}_{12} \hat{\Psi}\right)^{2}\right\} .
\end{aligned}
$$


The change of basis (4.6) causes a permutation among the matrices $\mathbf{X}_{\mu \rho}$ with $\mu, \rho=0,1,2,3$. Hamiltonians (4.7a) or (4.7b) are to be normal ordered by using point-splitting and Wick's theorem. These normal-ordered Hamiltonians are then bosonized by using the identities

$$
\hat{\psi}_{\mathrm{R}}^{\dagger}(x)=: \hat{\eta}_{\mathrm{R}} \frac{e^{-\mathrm{i} \hat{\varphi}_{\mathrm{R}}(x)}}{\sqrt{2 \pi \epsilon}}, \quad \hat{\psi}_{\mathrm{L}}^{\dagger}(x)=: \hat{\eta}_{\mathrm{L}} \frac{e^{+\mathrm{i} \hat{\varphi}_{\mathrm{L}}(x)}}{\sqrt{2 \pi \epsilon}},
$$

where $\epsilon$ is a short-distance cutoff. Hereby, we defined the chiral bosonic fields that obey the algebra

$$
\begin{aligned}
{\left[\hat{\varphi}_{\mathrm{R}}(x), \hat{\varphi}_{\mathrm{R}}\left(x^{\prime}\right)\right] } & =-\left[\hat{\varphi}_{\mathrm{L}}(x), \hat{\varphi}_{\mathrm{L}}\left(x^{\prime}\right)\right] \\
& =\mathrm{i} \pi \operatorname{sgn}\left(x-x^{\prime}\right), \\
{\left[\hat{\varphi}_{\mathrm{R}}(x), \hat{\varphi}_{\mathrm{L}}\left(x^{\prime}\right)\right] } & =0,
\end{aligned}
$$

and Klein factors $\hat{\eta}_{\mathrm{R} / \mathrm{L}}$ that obey the algebra

$$
\left\{\hat{\eta}_{\mathrm{R}}, \hat{\eta}_{\mathrm{R}}\right\}=\left\{\hat{\eta}_{\mathrm{L}}, \hat{\eta}_{\mathrm{L}}\right\}=2, \quad\left\{\hat{\eta}_{\mathrm{R}}, \hat{\eta}_{\mathrm{L}}\right\}=0 .
$$

Hamiltonian (4.7a) has the bosonic representation

$$
\begin{aligned}
\widehat{H}_{\mathrm{bd} a}=\int & d x\left\{\frac{1}{2 \pi}\left[\left(\partial_{x} \hat{\varphi}_{\mathrm{L}}\right)^{2}+\left(\partial_{x} \hat{\varphi}_{\mathrm{R}}\right)^{2}\right]\right. \\
& +\frac{\left(\lambda_{1, a}^{2}+\lambda_{2, a}^{2}\right)}{\pi^{2}}\left(\partial_{x} \hat{\varphi}_{\mathrm{L}}+\partial_{x} \hat{\varphi}_{\mathrm{R}}\right)^{2} \\
& \left.+\frac{2\left(\lambda_{1, a}^{2}-\lambda_{2, a}^{2}\right)}{\pi^{2} \epsilon^{2}} \cos \left(2 \hat{\varphi}_{\mathrm{L}}+2 \hat{\varphi}_{\mathrm{R}}\right)\right\} .
\end{aligned}
$$

Hamiltonian (4.7b) has the bosonic representation

$$
\begin{aligned}
\widehat{H}_{\mathrm{bd} b}=\int & d x\left\{\frac{1}{2 \pi}\left[\left(\partial_{x} \hat{\varphi}_{\mathrm{L}}\right)^{2}+\left(\partial_{x} \hat{\varphi}_{\mathrm{R}}\right)^{2}\right]\right. \\
& +\frac{\left(\lambda_{1, b}^{2}+\lambda_{2, b}^{2}\right)}{\pi^{2}}\left(\partial_{x} \hat{\varphi}_{\mathrm{L}}-\partial_{x} \hat{\varphi}_{\mathrm{R}}\right)^{2} \\
& \left.+\frac{2\left(\lambda_{1, b}^{2}-\lambda_{2, b}^{2}\right)}{\pi^{2} \epsilon^{2}} \cos \left(2 \hat{\varphi}_{\mathrm{L}}-2 \hat{\varphi}_{\mathrm{R}}\right)\right\} .
\end{aligned}
$$

In Hamiltonians (4.9), we have removed the Klein factors by diagonalizing the operator $i \hat{\eta}_{\mathrm{R}} \hat{\eta}_{\mathrm{L}}$ and choosing the eigenvalue +1 sector in the Klein Hilbert space. The difference between the two sets $\mathcal{B}_{a}$ and $\mathcal{B}_{b}$ in (4.4) manifests itself as the sign with which $\hat{\varphi}_{\mathrm{R}}$ enters Hamiltonians (4.9a) and (4.9b), respectively. In Hamiltonian (4.9a), the cosine results from the squares of the backward-scattering term $\propto \hat{\psi}_{\mathrm{R}}^{\dagger} \hat{\psi}_{\mathrm{L}}+$ H.c. In Hamiltonian (4.9b), the cosine results from the squares of the backward-pairing terms $\propto \hat{\psi}_{\mathrm{R}}^{\dagger} \hat{\psi}_{\mathrm{L}}^{\dagger}+$ H.c. In the $\mathrm{O}(2)$ symmetric case that is defined by the condition

$$
\lambda_{1, m}^{2}=\lambda_{2, m}^{2}, \quad m=a, b,
$$

both cosine interactions vanish and the theory remains gapless. Away from the $\mathrm{O}(2)$ symmetric point, the minima of the cosines are two-fold degenerate. If the cosines dominate over the kinetic energy, they open a gap with a two-fold degenerate manifold of ground states. Since the dependence on interaction strengths have the same form in Hamiltonians (4.9a) and (4.9b), the boundaries in the corresponding phase diagrams are identical. However, the phases they separate can be different whenever they break spontaneously distinct symmetries.

The transformation [46]

$$
\hat{\varphi}_{\mathrm{L}} \rightarrow+\hat{\varphi}_{\mathrm{L}}, \quad \hat{\varphi}_{\mathrm{R}} \rightarrow-\hat{\varphi}_{\mathrm{R}}
$$

that interchanges Hamiltonians (4.9a) and (4.9b) is nothing but the transformation that interchanges the pair of dual fields

$$
\begin{aligned}
& \hat{\phi}(x):=\frac{1}{\sqrt{4 \pi}}\left[\hat{\varphi}_{\mathrm{L}}(x)+\hat{\varphi}_{\mathrm{R}}(x)\right], \\
& \hat{\theta}(x):=\frac{1}{\sqrt{4 \pi}}\left[\hat{\varphi}_{\mathrm{L}}(x)-\hat{\varphi}_{\mathrm{R}}(x)\right],
\end{aligned}
$$

that satisfy the algebra

$$
\left[\hat{\phi}(x), \hat{\theta}\left(x^{\prime}\right)\right]=\frac{\mathrm{i}}{2} \operatorname{sgn}\left(x^{\prime}-x\right)
$$

with all other commutators vanishing. If one trades the Hamiltonian representation for the Lagrangian representation, one obtains the pair of actions

$$
\begin{aligned}
S_{a} & =\int d^{2} x\left\{\frac{1}{2 g_{a}}\left(\partial_{\mu} \phi\right)^{2}+\kappa_{a} \cos (\sqrt{16 \pi} \phi)\right\}, \\
S_{b} & :=\int d^{2} x\left\{\frac{1}{2 g_{b}}\left(\partial_{\mu} \theta\right)^{2}+\kappa_{b} \cos (\sqrt{16 \pi} \theta)\right\},
\end{aligned}
$$

where $\phi$ and $\theta$ are dual scalar fields satisfying either

$$
\partial_{\mu} \phi=\mathrm{i} g_{a} \epsilon_{\mu \nu} \partial_{\nu} \theta,
$$

with $\mu=0,1,\left(x_{0}, x_{1}\right)=\left(v_{a} \tau, x\right)$, or

$$
\partial_{\mu} \phi=\mathrm{i} g_{b} \epsilon_{\mu \nu} \partial_{\nu} \theta,
$$

with $\mu=0,1,\left(x_{0}, x_{1}\right)=\left(v_{b} \tau, x\right)$, respectively. The coupling constants are given by

$$
\begin{aligned}
& \frac{2}{v_{a}}=g_{a}:=\frac{1}{\sqrt{1+4 \frac{\lambda_{1, a}^{2}+\lambda_{2, a}^{2}}{\pi}}}, \\
& \frac{2}{v_{b}}=g_{b}:=\frac{1}{\sqrt{1+4 \frac{\lambda_{1, b}^{2}+\lambda_{2, b}^{2}}{\pi}}},
\end{aligned}
$$

whereas the effective interaction strengths are

$$
\begin{aligned}
& \kappa_{a}:=\frac{4}{\pi^{2} \epsilon^{2}} \sqrt{1+4 \frac{\lambda_{1, a}^{2}+\lambda_{2, a}^{2}}{\pi}}\left(\lambda_{1, a}^{2}-\lambda_{2, a}^{2}\right), \\
& \kappa_{b}:=\frac{4}{\pi^{2} \epsilon^{2}} \sqrt{1+4 \frac{\lambda_{1, b}^{2}+\lambda_{2, b}^{2}}{\pi}}\left(\lambda_{1, b}^{2}-\lambda_{2, b}^{2}\right) .
\end{aligned}
$$

The two actions (4.13a) and (4.13b) are exchanged if one performs the interchanges $\lambda_{i, a}^{2} \leftrightarrow \lambda_{i, b}^{2}$ with $i=1,2$ and $\phi \leftrightarrow$ $\theta$. The interaction strengths $(4.13 \mathrm{~g})$ and $(4.13 \mathrm{~h})$ change signs depending on whether $\lambda_{1, m}^{2}>\lambda_{2, m}^{2}$ or $\lambda_{1, m}^{2}<\lambda_{2, m}^{2}$, with $m=a, b$.

Before proceeding, we determine how the symmetries defined in Eqs. (2.3) act on the bosonic fields. The actions of the symmetry transformations on the complex fermionic fields are deduced from their actions on the Majorana fields and given 
by

$$
\begin{aligned}
& \widehat{U}_{\mathrm{C}}^{\dagger}\left(\begin{array}{c}
\hat{\psi}_{\mathrm{L}}(\tau, x) \\
\hat{\psi}_{\mathrm{R}}(\tau, x)
\end{array}\right) \widehat{U}_{\mathrm{C}}=\left(\begin{array}{c}
\hat{\psi}_{\mathrm{L}}(\tau, x) \\
\hat{\psi}_{\mathrm{R}}(\tau, x)
\end{array}\right), \\
& \widehat{U}_{\mathrm{T}}^{\dagger}\left(\begin{array}{c}
\hat{\psi}_{\mathrm{L}}(\tau, x) \\
\hat{\psi}_{\mathrm{R}}(\tau, x)
\end{array}\right) \widehat{U}_{\mathrm{T}}=\left(\begin{array}{c}
+\hat{\psi}_{\mathrm{R}}^{\dagger}(\tau, x) \\
-\hat{\psi}_{\mathrm{L}}^{\dagger}(\tau, x)
\end{array}\right), \\
& \widehat{U}_{\mathrm{R}}^{\dagger}\left(\begin{array}{c}
\hat{\psi}_{\mathrm{L}}(\tau, x) \\
\hat{\psi}_{\mathrm{R}}(\tau, x)
\end{array}\right) \widehat{U}_{\mathrm{R}}=\left(\begin{array}{l}
+\hat{\psi}_{\mathrm{R}}(\tau,-x) \\
-\hat{\psi}_{\mathrm{L}}(\tau,-x)
\end{array}\right),
\end{aligned}
$$

where $\widehat{U}_{\mathrm{C}}, \widehat{U}_{\mathrm{T}}$, and $\widehat{U}_{\mathrm{R}}$ are $\mathrm{PH}$, reversal of time, and reflection transformations at the many-body level. The operator $\widehat{U}_{\mathrm{T}}$ is defined to be antiunitary, whereas operators $\widehat{U}_{\mathrm{C}}$ and $\widehat{U}_{\mathrm{R}}$ are chosen to be unitary. We note that the PHS is represented by the identity, whereas the TRS involves a PH transformation [47]. These transformation laws together with Eqs. (4.8a) imply the transformation laws

$$
\begin{aligned}
& \widehat{U}_{\mathrm{C}}^{\dagger}\left(\begin{array}{c}
\hat{\varphi}_{\mathrm{L}}(\tau, x) \\
\hat{\varphi}_{\mathrm{R}}(\tau, x)
\end{array}\right) \widehat{U}_{\mathrm{C}}=\left(\begin{array}{c}
\hat{\varphi}_{\mathrm{L}}(\tau, x) \\
\hat{\varphi}_{\mathrm{R}}(\tau, x)
\end{array}\right), \\
& \widehat{U}_{\mathrm{T}}^{\dagger}\left(\begin{array}{c}
\hat{\varphi}_{\mathrm{L}}(\tau, x) \\
\hat{\varphi}_{\mathrm{R}}(\tau, x)
\end{array}\right) \widehat{U}_{\mathrm{T}}=\left(\begin{array}{c}
-\hat{\varphi}_{\mathrm{R}}(\tau, x) \\
-\hat{\varphi}_{\mathrm{L}}(\tau, x)+\pi
\end{array}\right), \\
& \widehat{U}_{\mathrm{R}}^{\dagger}\left(\begin{array}{c}
\hat{\varphi}_{\mathrm{L}}(\tau, x) \\
\hat{\varphi}_{\mathrm{R}}(\tau, x)
\end{array}\right) \widehat{U}_{\mathrm{R}}=\left(\begin{array}{c}
-\hat{\varphi}_{\mathrm{R}}(\tau,-x) \\
-\hat{\varphi}_{\mathrm{L}}(\tau,-x)
\end{array}\right) .
\end{aligned}
$$

We note that in deriving transformation rules (4.15), one must take care of the transformation rules on the Klein factors as well. Demanding the invariance of the operator $i \hat{\eta}_{R} \hat{\eta}_{L}$, we find the transformation rules

$$
\begin{aligned}
& \widehat{U}_{\mathrm{T}}^{\dagger}\left(\begin{array}{l}
\hat{\eta}_{\mathrm{L}} \\
\hat{\eta}_{\mathrm{R}}
\end{array}\right) \widehat{U}_{\mathrm{T}}=\left(\begin{array}{c}
+\hat{\eta}_{\mathrm{R}} \\
+\hat{\eta}_{\mathrm{L}}
\end{array}\right), \\
& \widehat{U}_{\mathrm{R}}^{\dagger}\left(\begin{array}{c}
\hat{\eta}_{\mathrm{L}} \\
\hat{\eta}_{\mathrm{R}}
\end{array}\right) \widehat{U}_{\mathrm{R}}=\left(\begin{array}{c}
+\hat{\eta}_{\mathrm{R}} \\
-\hat{\eta}_{\mathrm{L}}
\end{array}\right) .
\end{aligned}
$$

The corresponding transformation rules for the bosonic pair of dual fields are then found to be

$$
\begin{aligned}
& \widehat{U}_{\mathrm{C}}^{\dagger}\left(\begin{array}{c}
\hat{\phi}(\tau, x) \\
\hat{\theta}(\tau, x)
\end{array}\right) \widehat{U}_{\mathrm{C}}=\left(\begin{array}{c}
\hat{\phi}(\tau, x) \\
\hat{\theta}(\tau, x)
\end{array}\right), \\
& \widehat{U}_{\mathrm{T}}^{\dagger}\left(\begin{array}{c}
\hat{\phi}(\tau, x) \\
\hat{\theta}(\tau, x)
\end{array}\right) \widehat{U}_{\mathrm{T}}=\left(\begin{array}{c}
-\hat{\phi}(\tau, x)+\sqrt{\pi} / 2 \\
+\hat{\theta}(\tau, x)-\sqrt{\pi} / 2
\end{array}\right), \\
& \widehat{U}_{\mathrm{R}}^{\dagger}\left(\begin{array}{c}
\hat{\phi}(\tau, x) \\
\hat{\theta}(\tau, x)
\end{array}\right) \widehat{U}_{\mathrm{R}}=\left(\begin{array}{l}
-\hat{\phi}(\tau,-x) \\
+\hat{\theta}(\tau,-x)
\end{array}\right) .
\end{aligned}
$$

Alternatively, the transformations (4.17) can also be deduced from applying the many-body symmetry transformations on the components of the fermionic two-current.

Equipped with the transformation rules (4.17), we explore the phase diagram corresponding to the actions (4.13). For both actions, the corresponding cosine term has the scaling dimension

$$
\Delta_{m}:=\frac{4}{\sqrt{1+4 \frac{\lambda_{1, m}^{2}+\lambda_{2, m}^{2}}{\pi}}}, \quad m=a, b .
$$

Therefore, the cosine terms are IR irrelevant when $\lambda_{1, m}^{2}+$ $\lambda_{2, m}^{2}<3 \pi / 4$ and the theory remains critical. Increasing the interaction strengths makes the cosines relevant, in which case the fields $\theta$ and $\phi$ are pinned to the minima of the corresponding cosine terms in the ground state.

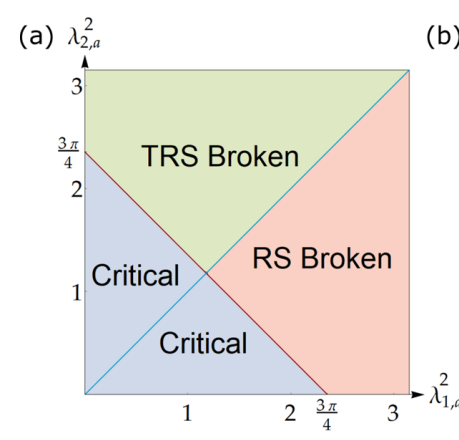

(b) $\lambda_{2, b}^{2}$

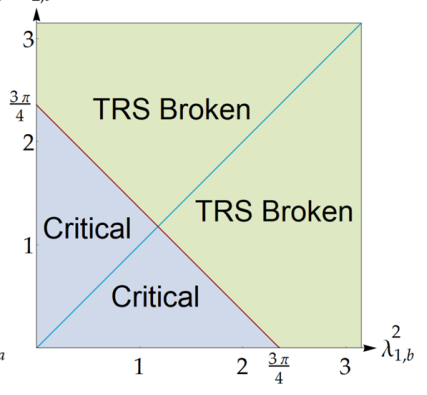

FIG. 1. Phase diagram for the edge theories defined by the actions (4.13a) $(m=a)$ in panel (a) and (4.13b) $(m=b)$ in panel (b) as a function of the interaction strengths $\lambda_{i, m}^{2}$ with $i=1,2$ and $m=a, b$. Along the blue line, $\mathrm{O}(2)$ symmetry holds and both cosine interactions vanish. Along the red line, both cosine interactions are marginal.

Each cosine has four extrema, two of which become minima depending on the difference $\lambda_{1, m}^{2}-\lambda_{2, m}^{2}$ being positive or negative. In particular, when this difference is zero, both cosines vanish and the low-energy effective theory is that of a free scalar field, i.e., it also remains critical. This is the $\mathrm{O}(2)$-symmetric line in the parameter space. Away from this line, we observe twofold ground-state degeneracy due to the two minima of the cosine.

For action (4.13a) with $\lambda_{1, a}^{2}>\lambda_{2, a}^{2}$, the two ground states are $\phi=\sqrt{\pi} / 4$ and $\phi=3 \sqrt{\pi} / 4$. The transformation rules (4.17) then imply that RS is spontaneously broken. Conversely, when $\lambda_{1, a}^{2}<\lambda_{2, a}^{2}$, the ground states correspond to $\phi=0$ and $\phi=\sqrt{\pi} / 2$, which implies that TRS is spontaneously broken.

For action (4.13b), the transformation rules (4.17) imply that RS always holds, whereas TRS is broken whenever there are two ground states separated by a shift of $\hat{\theta}$ by $\sqrt{\pi} / 2$. This is realized by the cosine interaction in (4.13b).

In Fig. 1, we plot the phase diagrams for both actions (4.13a) $(m=a)$ and (4.13b) $(m=b)$ as functions of the interaction strengths $\lambda_{1, m}^{2}$ and $\lambda_{2, m}^{2}$, respectively. For given $m=$ $a, b$, we define the red line in Fig. 1 by

$$
\lambda_{1, m}^{2}+\lambda_{2, m}^{2}=3 \pi / 4 \Longleftrightarrow \Delta_{m}=2
$$

and the blue line in Fig. 1 by

$$
\lambda_{1, m}^{2}=\lambda_{2, m}^{2}
$$

Below the red line (4.19), the cosine interactions are irrelevant as their scaling dimensions are larger than 2. Each point in coupling space is then a critical phase with algebraic correlation functions characterized by scaling exponents that are smooth functions of the couplings $\lambda_{1, m}^{2}$ and $\lambda_{2, m}^{2}$. The free Dirac point is defined by the origin $\lambda_{1, m}^{2}=\lambda_{2, m}^{2}=0$ of coupling space. Above the red line (4.19), the cosine interactions are relevant as their scaling dimensions are smaller than 2 . Each point in coupling space then belongs to a gapped phase, unless the couplings multiplying the cosine interactions vanish, as they do along the blue line (4.20). Each gapped phase is associated with a pattern of spontaneous symmetry breaking. When $\lambda_{1, a}^{2}<\lambda_{2, a}^{2}\left(\lambda_{1, a}^{2}>\lambda_{2, a}^{2}\right)$, TRS (RS) is spontaneously broken as follows from minimizing the cosine interaction. 
When $\lambda_{1, b}^{2} \neq \lambda_{2, b}^{2}$, TRS is spontaneously broken as follows again from minimizing the cosine interaction. Along the blue line (4.20), $\mathrm{O}(2)$ symmetry holds and both cosine interactions vanish. Along the red line, both cosine interactions are marginal.

Abelian bosonization reveals that when quartic contact interactions compatible with the DIIIR symmetries are added, gap opening necessarily breaks one of the defining symmetries. Therefore, the $v=2$ edge theory remains stable in the presence of interactions in the sense that it may only be gapped by interactions if any one of the protecting symmetries is either explicitly or spontaneously broken. We will next consider a generic family of symmetry-preserving cosine interactions and demonstrate that any interaction that gaps the edge theory must necessarily break spontaneously one of the protecting symmetries. We also discuss the effect of breaking of TS.

\section{B. Haldane criterion}

In Sec. IV A, we bosonized Hamiltonians (4.7). They are of the sine-Gordon type. In light of this result, one may consider a family of bosonic Hamiltonians with generic cosine interactions. These interactions can gap some, most, or all bosonic degrees of freedom. How many bosonic degrees of freedom remain gapless is determined using the so-called Haldane stability criterion [48]. Doing so in a manner compliant with imposing the protecting symmetries, we are going to recover the cosine potentials (4.9).

We consider the Hamiltonian

$$
\widehat{H}:=\widehat{H}_{0}+\widehat{H}_{\text {int }},
$$

which consists of the free Hamiltonian

$$
\widehat{H}_{0}:=\int d x \frac{1}{4 \pi}\left(\partial_{x} \widehat{\Phi}^{\top}\right)(x) V\left(\partial_{x} \widehat{\Phi}\right)(x),
$$

that describes free chiral bosonic fields and the interaction

$$
\widehat{H}_{\text {int }}:=-\int d x \sum_{T \in \mathbb{H}} h_{T}(x): \cos \left(T^{\top} K \widehat{\Phi}(x)+\alpha_{T}(x)\right):
$$

that encodes a countable set of local fermionic interactions describing many-body umklapp processes that we shall call tunneling processes and hence label with the symbol $T$. The components of the field $\widehat{\Phi}$ obey the commutation relations

$$
\left[\widehat{\Phi}_{i}(x), \widehat{\Phi}_{j}\left(x^{\prime}\right)\right]=-\mathrm{i} \pi\left[K_{i j}^{-1} \operatorname{sgn}\left(x-x^{\prime}\right)\right],
$$

where $K$ is a $2 \times 2$, integer valued, symmetric, and invertible matrix. The static functions

$$
h_{T}(x) \geqslant 0, \quad 0 \leqslant \alpha_{T}(x)<2 \pi
$$

encode the possibility that TS is broken on the edge. The matrix $V$ is a $2 \times 2$ symmetric and positive definite matrix. The two-dimensional tunneling vectors $T$ are chosen from a set $\mathbb{H}$, that we will specify later.

Our aim is to compare Hamiltonian (4.21a) with Hamiltonian (4.9a) or Hamiltonian (4.9b) and use the Haldane criterion to identify some minimal sets of tunneling vectors $\mathbb{H}$ that would gap the chiral bosonic fields $\widehat{\Phi}$ if the functions $h_{\mathrm{T}}$ were large. By comparing the free Hamiltonian (4.21b) with
(4.9), we define the fields

$$
\widehat{\Phi}(x):=\left(\begin{array}{ll}
\hat{\varphi}_{\mathrm{L}}(x) & \hat{\varphi}_{\mathrm{R}}(x)
\end{array}\right)^{\top},
$$

the universal data

$$
Q:=\left(\begin{array}{l}
1 \\
1
\end{array}\right), \quad K:=\left(\begin{array}{rr}
+1 & 0 \\
0 & -1
\end{array}\right)
$$

and the nonuniversal data

$$
V:=\left(\begin{array}{cc}
v & u \\
u & v
\end{array}\right), \quad 0<v \in \mathbb{R}, \quad 0 \leqslant u \in \mathbb{R} .
$$

With the universal data (4.22b), the algebra (4.21d) reduces to the algebra (4.8b). The two-dimensional vector $Q$ is the charge vector. The explicit dependence of the positive couplings $u$ and $v$ on the couplings $\lambda_{i, m}^{2}, i=1,2, m=a, b$ from Hamiltonian (4.9a) will not be needed in the following.

The minimal set of tunneling vectors $\mathbb{H}$ is defined as follows. We first construct the maximal Haldane set

$$
\mathbb{L}:=\left\{T \in \mathbb{Z}^{2} \mid T^{\top} K T^{\prime}=T^{\prime \top} K T=0, \forall T^{\prime} \in \mathbb{L}\right\},
$$

i.e., the set of elements in $\mathbb{Z}^{2}=\mathbb{Z} \times \mathbb{Z}$ such that the bilinear form $T^{\top} K T^{\prime}$ vanishes for any pair $T$ and $T^{\prime}$ from $\mathbb{L}$. This constraint is the compatibility condition of the Haldane criterion. With it, there is no competition between any pair of cosine interaction entering $\widehat{H}_{\text {int }}$. The vectors $T \in \mathbb{L}$ form a lattice since, for any pair $T, T^{\prime} \in \mathbb{L}$, the linear combination $n T+n^{\prime} T^{\prime}$ with $n, n^{\prime} \in \mathbb{Z}$ also satisfies the compatibility condition. We then define the minimal set of tunneling vectors as the subset $\mathbb{H} \subset \mathbb{L}$ such that elements $T \in \mathbb{H}$ constitutes the primitive cell of the lattice $\mathbb{L}$ which is compatible with the symmetry requirements of class DIIIR.

The Haldane criterion then asserts that the Hamiltonian (4.21c) for a given $\mathbb{H}$ removes $2 \times|\mathbb{H}|$-chiral bosonic fields from gapless degrees of freedom by pinning them (the notation $|\mathbb{H}|$ denotes the cardinality of the set $\mathbb{H})$. In our case, Hamiltonian (4.21a) consists of only a single pair of chiral bosonic fields. Therefore, it is enough to find the single tunneling vector making up $\mathbb{H}$ to remove all gapless degrees of freedom.

For a general tunneling vector $T=(m, n)$ of integers $m, n \in \mathbb{Z}$, the Haldane compatibility condition implies that there are two solutions, $n=m$ and $n=-m$. Therefore, there exists two disjoint sets of lattices $\mathbb{L}$ generated by the primitive cells

$$
\begin{aligned}
& \mathbb{H}_{a}:=\left\{\left(+n_{a}-n_{a}\right)^{\top} \mid n_{a} \text { to be determined }\right\}, \\
& \mathbb{H}_{b}:=\left\{\left(+n_{b}+n_{b}\right)^{\top} \mid n_{b} \text { to be determined }\right\} .
\end{aligned}
$$

The integers $n_{a}$ and $n_{b}$ are not yet determined. To determine how integers $n_{a}$ and $n_{b}$ are constrained, we define the pair of interactions

$$
\widehat{H}_{\mathrm{int} a}:=\int d x h_{a}(x): \cos \left(n_{a}\left[\hat{\varphi}_{\mathrm{L}}+\hat{\varphi}_{\mathrm{R}}\right](x)+\alpha_{a}(x)\right):
$$

and

$$
\widehat{H}_{\text {int } b}:=\int d x h_{b}(x): \cos \left(n_{b}\left[\hat{\varphi}_{\mathrm{L}}-\hat{\varphi}_{\mathrm{R}}\right](x)+\alpha_{b}(x)\right):,
$$

corresponding to the minimal sets (4.24a) and (4.24b), respectively, on which we shall impose the symmetries under 
the transformations defined in Eq. (4.17). Observe that, in the strong coupling limit

$$
4 \pi \sup \left\{h_{a}(x)\right\} \gg \max \{u, v\}
$$

[recall that $h_{a}(x) \geqslant 0$ for any $x$ and $u$ and $v$ are defined in the velocity matrix (4.22c)], the linear combinations $\hat{\varphi}_{\mathrm{L}}(x) \pm$ $\hat{\varphi}_{\mathrm{R}}(x)$ of the chiral fields are pinned to the minima of the cosine potentials, namely, either

$$
n_{a}\left[\hat{\varphi}_{\mathrm{L}}(x)+\hat{\varphi}_{\mathrm{R}}(x)\right]=2 \pi k+\pi-\alpha_{a}(x)
$$

or

$$
n_{b}\left[\hat{\varphi}_{\mathrm{L}}(x)-\hat{\varphi}_{\mathrm{R}}(x)\right]=2 \pi k+\pi-\alpha_{b}(x),
$$

respectively, for some integer $k \in \mathbb{Z}$.

\section{Symmetry constraints on Hamiltonian (4.25a)}

PHS is trivially satisfied by construction. Imposing TRS by using the transformation rule (4.15b) leads to the constraint

$$
\alpha_{a}(x)=-\alpha_{a}(x)-n_{a} \pi \bmod 2 \pi,
$$

which implies

$$
\alpha_{a}(x)=l_{a} \pi-\frac{n_{a} \pi}{2} \bmod 2 \pi, \quad l_{a}=0,1,
$$

since $\alpha_{a}(x) \in[0,2 \pi)$. Imposing RS by using the transformation rule $(4.15 \mathrm{c})$ leads to the constraint

$$
h_{a}(-x)=h_{a}(x), \quad \alpha_{a}(-x)=-\alpha_{a}(x) \bmod 2 \pi .
$$

Combining TRS and RS implies that

$$
h_{a}(x)=h_{a}(-x)
$$

and

$$
\alpha_{a}(x)=\left[f_{a}(|x|)-\frac{n_{a}}{2}\right] \pi \operatorname{sgn}(x) \bmod 2 \pi,
$$

where $f_{a}(x)$ is any function such that

$$
f_{a}:[0, \infty) \rightarrow\left\{l_{a}: l_{a}=0,1\right\} .
$$

We note that for any even $n_{a}$, assuming that $f_{a}(|x|)$ is constant, the discontinuity at $x=0$ of $\alpha_{a}(x)$ is an even multiple of $2 \pi$ so the solution to Eqs. (4.29b) and (4.29c) can be chosen independent of $x$. This is not the case for odd $n_{a}$ as $n_{a} \pi \operatorname{sgn}(x) / 2 \bmod 2 \pi$ changes by $\pi \bmod 2 \pi$ across $x=0$. A set of minima for the interaction (4.25a) compatible with TRS and RS that are labeled by the integers $l_{a}$ and $n_{a}$ are thus given by

$$
n_{a}\left[\hat{\varphi}_{\mathrm{L}}(x)+\hat{\varphi}_{\mathrm{R}}(x)\right]+\left(l_{a}-\frac{n_{a}}{2}\right) \pi \operatorname{sgn}(x)=\pi,
$$

where the right-hand side is defined modulo $2 \pi$. Here, to minimize the cost in kinetic energy arising from discontinuities, we restrict discontinuities to occur only at $x=0$ and demand that $h(x)$ vanishes smoothly at $x=0$ if the argument of the cosine is discontinuous at $x=0$. From now on, we only consider the cases $n_{a}=1$ and $n_{a}=2$.

When $n_{a}=1$, the minima (4.29d) simplify to

$$
\begin{aligned}
\hat{\varphi}_{\mathrm{L}}(x)+\hat{\varphi}_{\mathrm{R}}(x) & =\pi+\left(1 / 2-l_{a}\right) \pi \operatorname{sgn}(x) \bmod 2 \pi \\
& = \begin{cases}\frac{\pi}{2} \operatorname{sgn}(-x), & \text { if } l_{a}=0 \\
\frac{\pi}{2} \operatorname{sgn}(x), & \text { if } l_{a}=1 .\end{cases}
\end{aligned}
$$

One verifies that

$$
\left[\hat{\varphi}_{\mathrm{L}}(x)+\hat{\varphi}_{\mathrm{R}}(x)\right]^{\prime}= \begin{cases}\frac{\pi}{2} \operatorname{sgn}(-x), & \text { if } l_{a}=0 \\ \frac{\pi}{2} \operatorname{sgn}(x), & \text { if } l_{a}=1,\end{cases}
$$

where the prime over the operators on the left-hand side is a short-hand notation for their image under either reversal of time or the reflection as defined by Eq. (4.15). Therefore, for a given phase profile specified by $l_{a}$, there exists a unique gapped ground state for the bosonic interaction $\widehat{H}_{\text {int } a}$ that is invariant under the action of either TRS or RS. When $n_{a}=1$ and the competition between the kinetic energy and the interaction (4.25a) results in the opening of a spectral gap (with a midgap bound state) on the edge, TRS and RS are neither broken explicitly nor spontaneously, while TS is explicitly broken. As announced below Eqs. (2.6) by making use of the bulk-edge correspondence, the noninteracting topological classification $\mathbb{Z}$ of symmetry class DIIIR in $(2+1)$-dimensional space-time reduces to the topological classification $\mathbb{Z}_{2}$ of symmetry class DIII when a RS compliant breaking of TS is allowed [9,22], since $\widehat{H}_{\text {int } a}$ with $n_{a}=1$ is nothing but a fermionic mass term in the complex fermion representation. The midgap states bound at the reflection symmetric points are protected by the actions of TRS and RS and cannot be gapped. Such protected corner modes are nothing but the signature of a second-order SPT phase induced by the spatially varying mass term. Indeed, it has been shown in Ref. [49] that a two-dimensional superconductor in the symmetry class DIII with RS but no TS along the boundary is an example of a second-order SPT phase [50].

When $n_{a}=2$, the minima (4.29d) simplify to

$2\left[\hat{\varphi}_{\mathrm{L}}(x)+\hat{\varphi}_{\mathrm{R}}(x)\right]=\pi+\left(1-l_{a}\right) \pi \operatorname{sgn}(x) \bmod 2 \pi$.

Because

$$
\pi \operatorname{sgn}(x)=\pi \bmod 2 \pi, \quad-\pi=\pi \bmod 2 \pi,
$$

one may write

$$
2\left[\hat{\varphi}_{\mathrm{L}}(x)+\hat{\varphi}_{\mathrm{R}}(x)\right]=\pi l_{a}, \bmod 2 \pi .
$$

We conclude that

$$
\hat{\varphi}_{\mathrm{L}}(x)+\hat{\varphi}_{\mathrm{R}}(x)= \begin{cases}0, & \text { if } l_{a}=0 \\ \pi, & \text { if } l_{a}=0 \\ \pi / 2, & \text { if } l_{a}=1 \\ 3 \pi / 2, & \text { if } l_{a}=1 .\end{cases}
$$

One verifies that

$$
\left[\hat{\varphi}_{\mathrm{L}}(x)+\hat{\varphi}_{\mathrm{R}}(x)\right]_{\mathrm{TRS}}= \begin{cases}\pi, & \text { if } l_{a}=0 \\ 0, & \text { if } l_{a}=0 \\ \pi / 2, & \text { if } l_{a}=1 \\ 3 \pi / 2, & \text { if } l_{a}=1\end{cases}
$$

and

$$
\left[\hat{\varphi}_{\mathrm{L}}(x)+\hat{\varphi}_{\mathrm{R}}(x)\right]_{\mathrm{RS}}= \begin{cases}0, & \text { if } l_{a}=0 \\ \pi, & \text { if } l_{a}=0 \\ 3 \pi / 2, & \text { if } l_{a}=1 \\ \pi / 2, & \text { if } l_{a}=1,\end{cases}
$$

where the subscripts TRS and RS are short-hand notations for the image of the minima under reversal of time and space inversion, respectively. There are two crucial differences between the cases $n_{a}=1$ and $n_{a}=2$. The minima (4.33a) 
transform in a nontrivial way under the actions of TRS and RS. For each choice $l_{a}$, two minima are exchanged under the action of either reversal of time or space inversion. Furthermore, the compactness of the chiral fields and the choice $n_{a}=$ 2 conspire in such a way that they minimize the interaction $\widehat{H}_{\text {int } a}$ without breaking the TS.

The cosine in the interaction $\widehat{H}_{\text {int } a}$ with $n_{a}=2$ is identical to the cosine in Hamiltonian (4.9a). The coupling $h(x) \geqslant 0$ breaks TS in the interaction $\widehat{H}_{\text {int } a}$ when it is not a constant function of $x$, unlike the coupling that multiplies the cosine in Hamiltonian (4.9a). The two choices for $l_{a}$ in Eq. (4.33a) correspond to fixing the overall sign of the interaction $\widehat{H}_{\mathrm{int}} a$ with $n_{a}=2$ when evaluated at its translation symmetric minima. In other words, the two choices for $l_{a}$ in Eq. (4.33a) with $n_{a}=2$ correspond to choosing which two translation symmetric extrema of the cosine term are the minima. Furthermore, from the transformation rules (4.33b) and (4.33c), we observe that the same patterns for spontaneous symmetry-breaking patterns as with Hamiltonian (4.9a). When $l_{a}=0$, TRS is spontaneously broken, whereas RS is protected. When $l_{a}=$ 1 , RS is spontaneously broken, whereas TRS is protected. Hence, even though the interaction (4.25a) breaks TS when $h(x)$ is not a constant function of $x$, it shares with Hamiltonian (4.9a) the same phase diagram.

Finally, we note that the sign function that interpolates between any two translation symmetric minima of the interaction $\widehat{H}_{\text {int } a}$ also minimizes $\widehat{H}_{\text {int } a}$. One verifies that this sign function respects TRS and RS but breaks TS. Unlike the translation symmetric minima of the interaction $\widehat{H}_{\operatorname{int} a}$, this sign function costs kinetic energy. The competition between the kinetic and interaction terms results in a compromise by which the singularity of the sign function is smoothed. The outcome is a soliton that keeps TRS and RS but breaks TS. This soliton is a gapped excitation that can be interpreted as a pair of helical Majorana modes localized in the region where the soliton energy density is nonvanishing and whose existence is protected by TRS and RS in the Majorana representation of the boundary theory.

\section{Symmetry constraints on Hamiltonian (4.25b)}

PHS is again satisfied trivially by construction. Imposing TRS by using the transformation rule (4.17b) leads to the constraint

$$
n_{b}=2 m, \quad m \in \mathbb{Z},
$$

i.e., $n_{b}$ is an even integer. Imposing RS by using the transformation rule $(4.17 \mathrm{c})$ leads to the pair of constraints

$$
h_{b}(-x)=h_{b}(x), \quad \alpha_{b}(-x)=\alpha_{b}(x) .
$$

A set of minima is given by

$$
n_{b}\left[\hat{\varphi}_{\mathrm{L}}(x)-\hat{\varphi}_{\mathrm{R}}(x)\right]+\pi l_{b}=\pi, \quad \bmod 2 \pi,
$$

where $l_{b}=0,1$. We only consider the case $n_{b}=2$ and conclude that

$$
\hat{\varphi}_{\mathrm{L}}(x)-\hat{\varphi}_{\mathrm{R}}(x)= \begin{cases}\pi / 2, & \text { if } l_{b}=0 \\ 3 \pi / 2, & \text { if } l_{b}=0 \\ 0, & \text { if } l_{b}=1 \\ \pi, & \text { if } l_{b}=1\end{cases}
$$

One verifies that

$$
\left[\hat{\varphi}_{\mathrm{L}}(x)-\hat{\varphi}_{\mathrm{R}}(x)\right]_{\mathrm{TRS}}= \begin{cases}3 \pi / 2, & \text { if } l_{b}=0 \\ \pi / 2, & \text { if } l_{b}=0 \\ \pi, & \text { if } l_{b}=1 \\ 0, & \text { if } l_{b}=1\end{cases}
$$

The four translation symmetric minima (4.36) are invariant under the action of RS. On the other hand, under the action of TRS, two translation symmetric minima corresponding to each $l_{b}$ are exchanged. Therefore, RS is always protected by the interaction $\widehat{H}_{\text {int } b}$ with $n_{b}=2$, whereas TRS is spontaneously broken by its minima. The argument of the cosine in $\widehat{H}_{\text {int } b}$ with $n_{b}=2$ is identical to that of the cosine in Hamiltonian (4.9b). Hence, both Hamiltonians obey the same pattern of symmetry breaking. Finally, even though the interaction (4.25b) breaks TS when $h(x)$ is not a constant function of $x$, it shares with Hamiltonian (4.9b) the same phase diagram (Fig. 1).

\section{THE CASE $v=1$}

For the $v=1$ case, the boundary theory consists of a single helical pair of Majorana fields. In this case, as we shall explain, it is not possible to employ the gradient expansion method used in Sec. III. Instead, we proceed in two steps. First, we establish that there are two topological sectors in the effective bosonic theory for the boundary. Second, we write down the dominant quartic interaction which we treat within the mean-field approximation.

\section{A. Existence of two topological sectors}

The set (2.11) with $n=1$ has the 4 elements $\left(\mathrm{X}_{\mu} \equiv \sigma_{\mu}\right.$ with $\mu=0, \cdots 3)$. For $v=1$, there is at most $\mathrm{N}(1)=1$ interaction channel allowed by the symmetry conditions (2.6). Therefore, there is a unique parametrization

$$
\begin{aligned}
\mathcal{H}_{\mathrm{bd}}^{(\mathrm{dyn})}(\tau, x) & :=\beta_{0} \mathrm{i} \partial_{x}+\beta_{1} \phi(\tau, x), \\
\beta_{0} & :=\mathrm{X}_{3} \equiv \sigma_{3}, \quad \beta_{1}:=\mathrm{X}_{2} \equiv \sigma_{2}
\end{aligned}
$$

of the dynamical boundary single-particle Hamiltonian. If we impose the nonlinear constraint

$$
\phi^{2}(\tau, x) \equiv \bar{\phi}^{2}
$$

for some given real-valued number $\bar{\phi}$, the target manifold is then nothing but two points \pm 1 with the only nonvanishing homotopy group $\pi_{0}\left(\mathrm{~S}^{0}\right)=\mathbb{Z}_{2}$.

When the hard nonlinear constraint (5.3) is strictly imposed, all configurations of $\phi(\tau, x)$ other than the constant field $\phi(\tau, x)= \pm \bar{\phi}$ must be discontinuous at the space-time points where $\phi(\tau, x)$ switches between $+\bar{\phi}$ and $-\bar{\phi}$. The gradient of $\phi(\tau, x)$ is then ill-defined at singular points and zero everywhere else. If we relax the condition (5.3) by imposing the nonlinear constraint asymptotically,

$$
\lim _{\tau \rightarrow \pm \infty} \phi^{2}(\tau, x) \equiv \bar{\phi}^{2},
$$

then smooth deformations of these singular configurations are admissible. However, the continuous function $\phi(\tau, x)$ then necessarily takes the value zero along at least one time slice in $(1+1)$-dimensional space-time, which binds zero modes in 
the spectrum. This prevents employing the gradient expansion approach outlined in Sec. III since the Pfaffian obtained by integrating out real-valued Grassmann fields,

$$
\begin{aligned}
Z_{\mathrm{bd}} & \propto \int \mathcal{D}[\phi] \int \mathcal{D}[\chi] e^{-\int d^{2} x \bar{\chi}\left(\mathrm{i} \gamma_{\mu} \partial_{\mu}-\mathrm{i} \phi\right) \chi} \\
& \propto \int \mathcal{D}[\phi] \operatorname{Pf}\left[\mathrm{i} \sigma_{2} D[\phi]\right],
\end{aligned}
$$

vanishes due to zero eigenvalues of the kernel

$$
D:=\mathrm{i} \gamma_{\mu} \partial_{\mu}-\mathrm{i} \phi, \quad \gamma_{0}:=-\sigma_{2}, \quad \gamma_{1}:=\sigma_{1},
$$

where $\bar{\chi}=\chi^{\dagger}\left(\mathrm{i} \sigma_{2}\right)$. Because the kernel $\mathrm{i} \sigma_{2} D$ is skew symmetric, the identity

$$
\left(\operatorname{Pf}\left[\mathrm{i} \sigma_{2} D[\phi]\right]\right)^{2}=\operatorname{Det}\left[\mathrm{i} \sigma_{2} D[\phi]\right]
$$

holds. Therefore, the Pfaffian of $i \sigma_{2} D$, is nothing but the square root of the functional determinant of $i \sigma_{2} D$.

The idea that we shall develop below is the following. According to Eq. (5.6), computing the Pfaffian of a skewsymmetric operator is akin to taking the square root of a number. Taking the square root of a real-valued number yields two roots differing by their signs. For any pair $\phi$ and $\phi^{\prime}$, it is the relative sign between $\operatorname{Pf}\left[i \sigma_{2} D[\phi]\right]$ and $\operatorname{Pf}\left[i \sigma_{2} D\left[\phi^{\prime}\right]\right]$ that fixes if $\phi$ is topologically equivalent to $\phi^{\prime}$. The background $\phi$ is topologically equivalent to $\phi^{\prime}$ if

$$
\operatorname{sgn}\left(\frac{\operatorname{Pf}\left[\mathrm{i} \sigma_{2} D[\phi]\right]}{\operatorname{Pf}\left[\mathrm{i} \sigma_{2} D\left[\phi^{\prime}\right]\right]}\right)=+1 .
$$

Otherwise, the background $\phi$ is not topologically equivalent to $\phi^{\prime}$. We are going to show that there are two topological sectors in the effective bosonic theory, i.e., there are two disjoint sets of topologically inequivalent profiles of the field $\phi$.

Although the kernel $\mathrm{i} \sigma_{2} D[\phi]$ is not Hermitian, the kernel

$$
\begin{aligned}
D^{\prime}[\phi] & :=\left(\begin{array}{ll}
-\phi & +\partial \\
+\bar{\partial} & +\phi
\end{array}\right)=-\mathrm{i} \sigma_{1} \partial_{x}+\mathrm{i} \sigma_{2} \partial_{\tau}-\sigma_{3} \phi, \\
\partial & :=\partial_{\tau}-\mathrm{i} \partial_{x}, \quad \bar{\partial}:=-\partial_{\tau}-\mathrm{i} \partial_{x}
\end{aligned}
$$

(i) shares the same determinant as $\mathrm{i} \sigma_{2} D[\phi]$ and (ii) is Hermitian. It follows that the eigenvalues of $D^{\prime}[\phi]$ are real valued. Moreover, the kernel $D^{\prime}[\phi]$ obeys the Bogoliubov-de Gennes condition and, hence, the nonvanishing real-valued eigenvalues of $D^{\prime}[\phi]$ come in pairs of opposite signs. We shall assume that all eigenvalues of $D^{\prime}[\phi]$ are nonvanishing. The label $\iota$ enumerates all pairs of eigenvalues $\pm\left|\lambda_{\iota}^{\prime}\right| \in \mathbb{R} \backslash\{0\}$ of $D^{\prime}[\phi]$. We then have the definition

$$
\operatorname{Pf}\left[\mathrm{i} \sigma_{2} D[\phi]\right]:=\prod_{\iota}\left|\lambda_{l}^{\prime}\right|
$$

that consists of choosing all the positive representatives of the pairs of nonvanishing eigenvalues. The question that immediately arises is if this definition can be done consistently over the entire target space of $\phi$. If the answer to this question is positive, then the target space is topologically trivial. Otherwise, it is not.

Our goal is to show that there are two distinct topological sectors as discussed above. To this end, we shall choose an arbitrary profile $\phi(\tau, x)$ that obeys the boundary conditions (5.4) and prove the identities

$$
\operatorname{sgn}\left(\frac{\operatorname{Pf}\left[\mathrm{i} \sigma_{2} D[\phi]\right]}{\operatorname{Pf}\left[\mathrm{i} \sigma_{2} D[\bar{\phi}]\right]}\right)=-\operatorname{sgn}\left(\frac{\operatorname{Pf}\left[\mathrm{i} \sigma_{2} D[\phi]\right]}{\operatorname{Pf}\left[\mathrm{i} \sigma_{2} D[-\bar{\phi}]\right]}\right)
$$

and

$$
\operatorname{sgn}\left(\frac{\operatorname{Pf}\left[i \sigma_{2} D[\phi]\right]}{\operatorname{Pf}\left[i \sigma_{2} D[-\phi]\right]}\right)=-1 .
$$

Two comments are in order before we prove Eqs. (5.10). Equation (5.10a) implies that profile $\phi$ is topologically equivalent to either one of the two constant profiles $\pm \bar{\phi}$. In other words, there exist exactly two topological sectors with representative profiles $+\bar{\phi}$ and $-\bar{\phi}$ as measured by Eq. (5.10a). Equation (5.10b) implies that the profiles $\phi$ and $-\phi$ belong to distinct topological sectors, a fact that originates from a $\mathbb{Z}_{2}$ global anomaly [51,52]. Indeed, the transformation

$$
\chi=\sigma_{3} \chi^{\prime}, \quad \phi=-\phi^{\prime}
$$

leaves the Lagrangian

$$
\bar{\chi}\left(\mathrm{i} \gamma_{\mu} \partial_{\mu}-\mathrm{i} \phi\right) \chi=\bar{\chi}^{\prime}\left(\mathrm{i} \gamma_{\mu} \partial_{\mu}-\mathrm{i} \phi^{\prime}\right) \chi^{\prime}
$$

invariant, while the partition function (5.5a) changes according to

$$
\begin{aligned}
Z_{\mathrm{bd}} & \propto \int \mathcal{D}\left[\phi^{\prime}\right] \mathcal{D}\left[\chi^{\prime}\right] \mathcal{J}\left[\sigma_{3}\right] e^{-\int d^{2} x \bar{\chi}^{\prime}\left(\mathrm{i} \gamma_{\mu} \partial_{\mu}-\mathrm{i} \phi^{\prime}\right) \chi^{\prime}} \\
& \propto \int \mathcal{D}\left[\phi^{\prime}\right] \mathcal{J}\left[\sigma_{3}\right] \operatorname{Pf}\left[\mathrm{i} \sigma_{2} D\left[\phi^{\prime}\right]\right] \\
& \propto \int \mathcal{D}[\phi] \mathcal{J}\left[\sigma_{3}\right] \operatorname{Pf}\left[\mathrm{i} \sigma_{2} D[-\phi]\right] .
\end{aligned}
$$

On the one hand, to reach the right-hand side of the second line, we allowed for a possibly nontrivial Jacobian $\mathcal{J}\left[\sigma_{3}\right]$ associated with the transformation $\chi=\sigma_{3} \chi^{\prime}$. On the other hand, to reach the third line, we assumed that the Jacobian associated with the transformation $\phi=-\phi^{\prime}$ is unity. Equation (5.10b) then implies that $\mathcal{J}\left[\sigma_{3}\right]=-1$, which is the precise definition of a $\mathbb{Z}_{2}$ global anomaly, namely, the symmetry of the Lagrangian that is not respected by the measure.

\section{Proof of Eqs. (5.10)}

We now prove Eqs. (5.10). To examine whether two profiles $\phi_{\mathrm{i}}(\tau, x)$ and $\phi_{\mathrm{f}}(\tau, x)$ are topologically equivalent, we introduce a parameter $t \in[0,1]$ and define a continuous function $\phi_{t}(\tau, x)$ such that

$$
\phi_{t=0}(\tau, x)=\phi_{\mathrm{i}}(\tau, x), \quad \phi_{t=1}(\tau, x)=\phi_{\mathrm{f}}(\tau, x) .
$$

We choose the linear interpolation

$$
\phi_{t}(\tau, x):=(1-t) \phi_{\mathrm{i}}(\tau, x)+t \phi_{\mathrm{f}}(\tau, x) .
$$

We impose periodic boundary conditions in both $\tau$ and $x$,

$$
\phi\left(\tau, x+L_{x}\right)=\phi(\tau, x), \quad \phi\left(\tau+L_{\tau}, x\right)=\phi(\tau, x) .
$$

Hence, interpolation (5.12) also satisfies these boundary conditions. Boundary conditions (5.13) describe a compact space-time $\left(\mathrm{S}^{1} \times \mathrm{S}^{1}=\mathrm{T}^{2}\right)$. It follows that the spectrum of the kernel $D^{\prime}\left[\phi_{t}\right]$ defined in Eq. (5.8) is discrete. If one calculates the flow of eigenvalues $\lambda_{t, l}^{\prime}$ of the kernel $D^{\prime}\left[\phi_{t}\right]$ as a function 
of $t$, whenever there is a gap closing, i.e., at least one of the $\lambda_{t, l}^{\prime}$ is 0 , there is a $\pi$ phase change in the Pfaffian. Thus, an odd number of gap closings during the evolution from $t=0$ to $t=1$ means that the initial and final profiles belong to different topological sectors. We will prove Eqs. (5.10) by assuming that the number of gap closings is independent of the choice of the interpolation scheme, without calculating the actual number of gap closings explicitly.

We first examine a special case of Eq. (5.10a) for which $\phi(\tau, x)=+\bar{\phi}$. Consider the linear interpolation

$$
\phi_{t}^{+,-}:=(1-t) \bar{\phi}+t(-\bar{\phi})=(1-2 t) \bar{\phi} .
$$

For any $t \neq 1 / 2, \phi_{t}^{+,-}$contributes to the Kernel $D^{\prime}\left[\phi_{t \neq 1 / 2}^{+,-}\right]$as a constant nonvanishing mass term. Hence, the spectrum is gapped. This gap closes only at $t=1 / 2$, in which case the kernel $D^{\prime}\left[\phi_{t=1 / 2}^{+,-}\right]$is that of a free Majorana fermion. There exists only a single pair of zero eigenvalues that are labeled by reciprocal vector $(\omega, k)=(0,0)$. Therefore, we find that there is a single crossing between negative and positive eigenvalues of $D^{\prime}\left[\phi_{t}^{+,-}\right]$at $t=1 / 2$. It follows that in the special case $\phi(\tau, x)=+\bar{\phi}$, Eq. (5.10a) holds. For any profile $\phi(\tau, x)$, the manipulation

$$
\begin{aligned}
\operatorname{sgn}\left(\frac{\operatorname{Pf}\left[\mathrm{i} \sigma_{2} D[\phi]\right]}{\operatorname{Pf}\left[\mathrm{i} \sigma_{2} D[\bar{\phi}]\right]}\right)= & \operatorname{sgn}\left(\frac{\operatorname{Pf}\left[\mathrm{i} \sigma_{2} D[\phi]\right]}{\operatorname{Pf}\left[\mathrm{i} \sigma_{2} D[-\bar{\phi}]\right]}\right) \\
& \times \operatorname{sgn}\left(\frac{\operatorname{Pf}\left[\mathrm{i} \sigma_{2} D[-\bar{\phi}]\right]}{\operatorname{Pf}\left[\mathrm{i} \sigma_{2} D[\bar{\phi}]\right]}\right)
\end{aligned}
$$

then implies Eq. (5.10a). Observe that identity (5.15) is nothing but the interpolation

$$
\Phi_{t}^{+,-}:= \begin{cases}(1-2 t) \phi(\tau, x)-2 t \bar{\phi}, & \text { if } 0 \leqslant t<\frac{1}{2} \\ (2 t-2) \bar{\phi}+(2 t-1) \bar{\phi}, & \text { if } \frac{1}{2} \leqslant t \leqslant 1 .\end{cases}
$$

To show Eq. (5.10b), we note that for any $\phi(\tau, x)$,

$$
\sigma_{2} \mathrm{~K} D^{\prime}[\phi] \mathrm{K} \sigma_{2}=D^{\prime}[-\phi] .
$$

Hence, $D^{\prime}[\phi]$ and $D^{\prime}[-\phi]$ share the same eigenvalue spectrum. This implies that for the two interpolations

$$
\begin{aligned}
& \Phi_{t}^{+}:=(1-t) \bar{\phi}+t \phi(\tau, x), \\
& \Phi_{t}^{-}:=(1-t)(-\bar{\phi})+t(-\phi(\tau, x))=-\Phi_{t}^{+},
\end{aligned}
$$

$D^{\prime}\left[\Phi_{t}^{+}\right]$and $D^{\prime}\left[\Phi_{t}^{-}\right]$also share the same eigenvalue spectrum. Therefore, one can then show that

$$
\operatorname{sgn}\left(\frac{\operatorname{Pf}\left[\mathrm{i} \sigma_{2} D[\phi]\right]}{\operatorname{Pf}\left[\mathrm{i} \sigma_{2} D[\bar{\phi}]\right]}\right)=\operatorname{sgn}\left(\frac{\operatorname{Pf}\left[\mathrm{i} \sigma_{2} D[-\phi]\right]}{\operatorname{Pf}\left[\mathrm{i} \sigma_{2} D[-\bar{\phi}]\right]}\right),
$$

which after rearrangement gives

$$
\begin{aligned}
\operatorname{sgn}\left(\frac{\operatorname{Pf}\left[i \sigma_{2} D[\phi]\right]}{\operatorname{Pf}\left[i \sigma_{2} D[-\phi]\right]}\right) & =\operatorname{sgn}\left(\frac{\operatorname{Pf}\left[i \sigma_{2} D[\bar{\phi}]\right]}{\operatorname{Pf}\left[i \sigma_{2} D[-\bar{\phi}]\right]}\right) \\
& =-1 .
\end{aligned}
$$

Any profile $\phi(\tau, x)$ is topologically inequivalent to $-\phi(\tau, x)$, as claimed in Eq. (5.10b).

\section{B. Mean-field treatment of the interaction}

To complement the discussion in the previous subsection, we integrate over the bosonic field $\phi$ in action (5.5a) and de- rive the effective action for the Majorana fields $\hat{\chi}_{L}$ and $\hat{\chi}_{R}$. The single interaction term has the form $\hat{\chi}_{\mathrm{L}}(x) \hat{\chi}_{\mathrm{R}}(x) \hat{\chi}_{\mathrm{L}}(x+\epsilon)$ $\hat{\chi}_{\mathrm{R}}(x+\epsilon)$, where $\epsilon$ is a short-distance cutoff that implements point splitting. For weak coupling strength, this interaction term is irrelevant and the boundary remains gapless. In the limit of a strong interaction strength, a gap opens in the spectrum [53,54]. At the mean-field level, this gap corresponds to the bilinear $i \hat{\chi}_{L} \hat{\chi}_{R}$ acquiring a nonvanishing expectation value. This is equivalent to replacing the dynamical field $\phi(\tau, x)$ in action (5.5a) by the constant profiles $\pm \bar{\phi}$. Inserting the mean-field solution for the field $\phi(\tau, x)$ explicitly breaks the TRS since the term $\pm \mathrm{i} \bar{\phi} \hat{\chi}_{\mathrm{L}} \hat{\chi}_{\mathrm{R}}$ is odd under the transformation (2.3b). Gapping the boundary is only possible by spontaneously breaking TRS.

\section{CONCLUSION}

We have studied by nonperturbative means the stability of a two-dimensional crystalline topological superconductor in symmetry class DIIIR when perturbed by symmetrypreserving quartic contact interactions. Building on the fact that eight copies of helical pairs of edge modes are gapped by such interactions without symmetry breaking, we investigated the stability of $v=1,2,4$ copies of the helical pairs of edge theories in order to understand how these cases remain stable. For $v=4$ copies of edge modes, we identified four interacting channels and presented an analytical derivation of the low-energy effective action, which is a NLSM model supplemented by a WZ term. In $(1+1)$ dimensions, this action flows to that of a gapless theory. Hence, the interacting theory remains gapless. We then employed bosonization methods to study interactions between $v=2$ copies of helical pairs of edge modes. We found that there is a regime in coupling space for which interactions become relevant, but always at the cost of the spontaneous breaking of one of the two protecting symmetries, provided we impose translation symmetry on the edge. For the final case of a single helical pair of edge modes, although we were not able to bosonize the fermionic theory explicitly, we showed that there exist two topological sectors and a $\mathbb{Z}_{2}$ global anomaly. We instead analyzed the stability of the noninteracting edge states by using their Majorana representation and showed that the boundary can only be gapped at the cost of spontaneously breaking the TRS.

In two-dimensional space, the symmetry class BDIR corresponds to a TRS crystalline superconductor for which the operation of time reversal squares to +1 . Its noninteracting topological classification $\mathbb{Z}$ becomes the classification $\mathbb{Z}_{8}$ in the presence of symmetry-preserving contact quartic interactions [35]. Our approach would also apply to this case. The stability analysis of two-dimensional crystalline insulators with noninteracting topological classification $\mathbb{Z}$ can always be dealt with using Abelian bosonization techniques in combination with the Haldane criterion. However, Abelian bosonization techniques are not applicable to three-dimensional space. Instead, one relies on functional bosonization techniques based on the gradient expansion or on conjectured dualities. Detailed stability analysis of some two- and three-dimensional topological crystalline insulators can be found in Refs. [55-57]. 


\section{ACKNOWLEDGMENTS}

Ö.M.A. was supported by the Swiss National Science Foundation (SNSF) under Grant No. 200021 184637. J.-H.C. was supported by the SNSF through Grants No. 2000021 153648 and No. P2EZP2-184306. S.R. is supported by the Na- tional Science Foundation under Award No. DMR-2001181 and by a Simons Investigator Grant from the Simons Foundation (Award No. 566116). A.F. was supported by JSPS KAKENHI (Grant No. 19K03680) and JST CREST (Grant No. JPMJCR19T2).
[1] K. v. Klitzing, G. Dorda, and M. Pepper, Phys. Rev. Lett. 45, 494 (1980).

[2] A. P. Schnyder, S. Ryu, A. Furusaki, and A. W. W. Ludwig, Phys. Rev. B 78, 195125 (2008).

[3] S. Ryu, A. P. Schnyder, A. Furusaki, and A. W. W. Ludwig, New J. Phys. 12, 065010 (2010).

[4] A. Kitaev, AIP Conf. Proc. 1134, 22 (2009).

[5] The notion of a spectral gap only applies if translation symmetry holds. When translation symmetry is broken by a static random potential that respects the protecting symmetries and is weak relative to the spectral gap, all eigenstates within the gap are insulating. The boundary states that were gapless with translation symmetry along the boundary remain delocalized in the presence of the static disorder potential.

[6] L. Fu, Phys. Rev. Lett. 106, 106802 (2011).

[7] Y. Ando and L. Fu, Annu. Rev. Condens. Matter Phys. 6, 361 (2015).

[8] L. Fu and C. L. Kane, Phys. Rev. B 76, 045302 (2007).

[9] C.-K. Chiu, H. Yao, and S. Ryu, Phys. Rev. B 88, 075142 (2013).

[10] F. Zhang, C. L. Kane, and E. J. Mele, Phys. Rev. Lett. 111, 056403 (2013).

[11] H. Song, S.-J. Huang, L. Fu, and M. Hermele, Phys. Rev. X 7, 011020 (2017).

[12] M. Koshino, T. Morimoto, and M. Sato, Phys. Rev. B 90, 115207 (2014).

[13] C.-X. Liu, R.-X. Zhang, and B. K. VanLeeuwen, Phys. Rev. B 90, 085304 (2014).

[14] T. Morimoto and A. Furusaki, Phys. Rev. B 88, 125129 (2013).

[15] R.-J. Slager, A. Mesaros, V. Juricic, and J. Zaanen, Nat. Phys. 9, 98 (2013).

[16] C. Fang, M. J. Gilbert, and B. A. Bernevig, Phys. Rev. B 87, 035119 (2013).

[17] P. Jadaun, D. Xiao, Q. Niu, and S. K. Banerjee, Phys. Rev. B 88, 085110 (2013).

[18] K. Shiozaki and M. Sato, Phys. Rev. B 90, 165114 (2014).

[19] L. Trifunovic and P. Brouwer, Phys. Rev. B 96, 195109 (2017).

[20] L. Fidkowski and A. Kitaev, Phys. Rev. B 81, 134509 (2010).

[21] L. Fidkowski and A. Kitaev, Phys. Rev. B 83, 075103 (2011).

[22] H. Yao and S. Ryu, Phys. Rev. B 88, 064507 (2013).

[23] S. Ryu and S.-C. Zhang, Phys. Rev. B 85, 245132 (2012).

[24] X.-L. Qi, New J. Phys. 15, 065002 (2013).

[25] L. Fidkowski, X. Chen, and A. Vishwanath, Phys. Rev. X 3, 041016 (2013).

[26] M. A. Metlitski, L. Fidkowski, X. Chen, and A. Vishwanath, arXiv:1406.3032.

[27] C. Wang and T. Senthil, Phys. Rev. B 89, 195124 (2014).

[28] T. Morimoto, A. Furusaki, and C. Mudry, Phys. Rev. B 92, 125104 (2015)

[29] Y.-Z. You and C. Xu, Phys. Rev. B 90, 245120 (2014).
[30] A. Kapustin, R. Thorngren, A. Turzillo, and Z. Wang, J. High Energy Phys. 12 (2015) 052.

[31] C. Wang, A. C. Potter, and T. Senthil, Science 343, 629 (2014).

[32] E. Tang and X.-G. Wen, Phys. Rev. Lett. 109, 096403 (2012).

[33] R. Queiroz, E. Khalaf, and A. Stern, Phys. Rev. Lett. 117, 206405 (2016).

[34] D. S. Freed and M. J. Hopkins, arXiv:1604.06527.

[35] X.-Y. Song and A. P. Schnyder, Phys. Rev. B 95, 195108 (2017).

[36] In other words, any local topological obstruction implies either a gapless ground state or gapped but degenerate ground states in the thermodynamic limit. Conversely, a nondegenerate ground state in the thermodynamic limit implies no local topological obstruction. However, the absence of any local topological obstruction does not imply a nondegenerate ground state in the thermodynamic limit.

[37] A. Abanov and P. B. Wiegmann, Nucl. Phys. B 570, 685 (2000).

[38] Had we chosen a unitary Hermitian representation of reflection that realizes a different algebra with the time-reversal or the interchange of particles and holes, the classification would be either trivial or have a $\mathbb{Z}_{2}$ group structure.

[39] E. Witten, Commun. Math. Phys. 92, 455 (1984).

[40] T. Morimoto, A. Furusaki, and C. Mudry, Phys. Rev. B 91, 235111 (2015).

[41] A. Polyakov, Phys. Lett. B 59, 79 (1975).

[42] P. Hosur, S. Ryu, and A. Vishwanath, Phys. Rev. B 81, 045120 (2010).

[43] J. Wess and B. Zumino, Phys. Lett. B 37, 95 (1971).

[44] S. P. Novikov, Russ. Math. Surveys 37, 1 (1982).

[45] E. Witten, Nucl. Phys. B 223, 422 (1983).

[46] Recall that two copies of the helical Majorana fields can be thought of as a low-energy description of two copies of the Ising model. Suppose now that a Kramers-Wannier duality transformation is applied to only the second copy of the Ising model via the transformation $\hat{\chi}_{\mathrm{L}}^{2} \rightarrow \hat{\chi}_{\mathrm{L}}^{2}$ and $\hat{\chi}_{\mathrm{R}}^{2} \rightarrow-\hat{\chi}_{\mathrm{R}}^{2}$. In the language of complex fermions, the left-handed component $\hat{\psi}_{\mathrm{L}}$ is unchanged, while the right-handed component $\hat{\psi}_{\mathrm{R}}$ is transformed into its dagger, i.e., $\hat{\psi}_{\mathrm{R}} \rightarrow \hat{\psi}_{\mathrm{R}}^{\dagger}$. The transformation Eq. (4.11) of chiral bosons then follows.

[47] In fact, the unitary particle-hole transformation operator $\hat{U}_{\mathrm{C}}$ replaces the spinor $\hat{\Psi}$ with its conjugate transpose $\hat{\Psi}^{\dagger}(\tau, x)$ by the transformation rule $\hat{U}_{\mathrm{C}}^{\dagger} \hat{\Psi}(\tau, x) \hat{U}_{\mathrm{C}}=\hat{\Psi}^{\dagger}(\tau, x) \mathrm{M}$, where $\mathrm{M}$ is a unitary matrix. It follows from the Majorana reality condition Eq. (2.2b) and the representation Eq. (2.6a) that $M=X_{10}$. This implies the transformation rule Eq. (4.14a) for the individual components of the spinor $\hat{\Psi}(\tau, x)$.

[48] F. D. M. Haldane, Phys. Rev. Lett. 74, 2090 (1995).

[49] J. Langbehn, Y. Peng, L. Trifunovic, F. von Oppen, and P. W. Brouwer, Phys. Rev. Lett. 119, 246401 (2017).

[50] The topological index belongs to the group $\mathbb{Z}_{2}$. Hence, the midgap state bound by a dynamical mass supporting a domain 
wall for the $v=4$ case can be gapped as opposed to the $v=2$ case.

[51] E. Witten, Phys. Lett. B 117, 324 (1982).

[52] S. Ryu, C. Mudry, H. Obuse, and A. Furusaki, Phys. Rev. Lett. 99, 116601 (2007).

[53] D. Aasen, R. S. K. Mong, B. M. Hunt, D. Mandrus, and J. Alicea, Phys. Rev. X 10, 031014 (2020).

[54] Y.-Z. Chou and R. M. Nandkishore, Phys. Rev. B 103, 075120 (2021).
[55] T. Yoshida and A. Furusaki, Phys. Rev. B 92, 085114 (2015).

[56] H. Isobe and L. Fu, Phys. Rev. B 92, 081304(R) (2015).

[57] C.-T. Hsieh, G. Y. Cho, and S. Ryu, Phys. Rev. B 93, 075135 (2016).

Correction: Three sentences in the fifth paragraph of Sec. I were inadvertently duplicated during the production process and have been removed, as they appear in Endnote [36]. Equation (3.4a) contained a minor error and has been fixed. 